\title{
Asymptotic Analysis of Stock Price Densities and Implied Volatilities in Mixed Stochastic Models*
}

\author{
Archil Gulisashvili ${ }^{\dagger}$ and Josep Vives ${ }^{\ddagger}$
}

\begin{abstract}
In this paper, we obtain sharp asymptotic formulas with error estimates for the Mellin convolution of functions defined on $(0, \infty)$ and use these formulas to characterize the asymptotic behavior of marginal distribution densities of stock price processes in mixed stochastic models. Special examples of mixed models are jump-diffusion models and stochastic volatility models with jumps. We apply our general results to the Heston model with double exponential jumps and perform a detailed analysis of the asymptotic behavior of the stock price density, the call option pricing function, and the implied volatility in this model. We also obtain similar results for the Heston model with jumps distributed according to the normal inverse Gaussian law.
\end{abstract}

Key words. mixed stochastic stock price models, Mellin convolution, Heston model with double exponential jumps, implied volatility

AMS subject classifications. 91G20, 91B25, 34E05

DOI. $10.1137 / 140962255$

1. Introduction. The random behavior of the stock price in a mixed model is described by a stochastic process $X=X^{(1)} X^{(2)}$, where $X^{(1)}$ and $X^{(2)}$ are strictly positive independent integrable processes on a complete filtered probability space $\left(\Omega, \mathcal{F},\left\{\mathcal{F}_{t}\right\}, \mathbb{P}\right)$. Important examples of mixed models are jump-diffusion models and stochastic volatility models with Lévytype jumps. More information on models with jumps can be found in [11] and [26].

In this paper, we obtain asymptotic formulas with error estimates for the distribution density of the stock price and the implied volatility in special mixed stochastic stock price models. Let us suppose that the distributions $\mu_{t}^{(1)}$ and $\mu_{t}^{(2)}$ of the random variables $X_{t}^{(1)}$ and $X_{t}^{(2)}$ in a mixed stochastic model admit densities $D_{t}^{(1)}$ and $D_{t}^{(2)}$, respectively. Then the distribution $\mu_{t}$ of the stock price $X_{t}$ also admits density $D_{t}$, which can be represented by the Mellin convolution

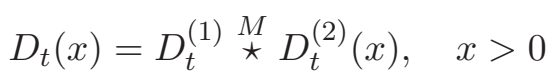

(the definition of the Mellin convolution is given below). The fact that the distribution density of the product of two independent random variables is the Mellin convolution of their densities was mentioned in [12].

In [5] (see also [9]), Arandelović obtained an asymptotic formula for the Mellin convolution of functions defined on the half-line $(0, \infty)$. However, Arandelović's formula does not contain

\footnotetext{
* Received by the editors March 25, 2014; accepted for publication (in revised form) December 29, 2014; published electronically March 18, 2015. This research was partially supported by Spanish grant MEC MTM 201231192.

http://www.siam.org/journals/sifin/6/96225.html

${ }^{\dagger}$ Department of Mathematics, Ohio University, Athens, OH 45701 (gulisash@ohio.edu).

‡Departament de Probabilitat, Lògica i Estadística, Universitat de Barcelona, 08007-Barcelona (Catalunya), Spain (josep.vives@ub.edu).
} 
an error estimate. In subsection 2.3 of the present paper, asymptotic formulas with error estimates are established for the Mellin convolution (see the formulas in Theorems 8 and 9). These formulas extend Arandelović's result. They are used in the paper to characterize the asymptotic behavior of the stock price density in special mixed stochastic stock price models. Asymptotic expansions of the Mellin convolution under different restrictions than those imposed in Arandelović's work and in the present paper were obtained by Handelsman and Lew (see the presentation of their results in section 3.4 of Wong [27]).

One of the examples considered below is the Heston model with asymmetric double exponential jumps. Theorems 10 and 11 obtained in this paper deal with the case where the jump part of the mixed model dominates, while Theorems 12 and 13 concern the asymptotics of the stock price density in the case where the Heston part dominates. Weaker estimates were obtained earlier in [19]. In section 6, we briefly discuss some other models.

In [22] (see also [23]), Kou introduced and studied a jump-diffusion model that is in fact a mixture of the Black-Scholes model with the double exponential jump model. An asymptotic formula (without an error estimate) for the distribution function of the stock price in the Kou model was obtained in [2, Example 7.6]. In [29] and [15], an asymptotic formula with an error estimate was found for the call pricing function in the Kou model. In the present paper, we obtain a similar formula for a class of models, including the Heston model with double exponential jumps and the Kou model (see (45)). In our opinion the error estimate in formula (45) is better than that in [15].

In our analysis of the stock price density in the Heston model perturbed by double exponential jumps, we use some of the results obtained in [22]. It is interesting that the asymptotic behavior of the stock price density in the Heston model without jumps and that of the absolutely continuous part of the distribution associated with the double exponential jump part are similar (compare (32) with (93) and (33) with (94)). It follows that in the study of the asymptotic behavior of the stock price density in the mixture of the Heston model with the double exponential jump model, we have to take into account which part of the mixed model dominates the other. This dichotomy does not appear in the Kou model since the double exponential jump part always dominates the Black-Scholes part. A certain similarity between the asymptotic behavior of the call pricing functions in the Heston model without jumps and that in the Kou model was observed in [15] too.

Asymptotic formulas for the stock price density can be used to study the asymptotic behavior of option pricing functions and the implied volatility. In section 4 of the present paper, we obtain asymptotic formulas with five explicit terms and error estimates for the implied volatility at extreme strikes in the Heston model with double exponential jumps. Similar formulas for the Heston model with normal inverse Gaussian (NIG) type jumps are established in section 6. We use some of the methods developed in [14] and [17] to estimate the implied volatility. Slightly weaker asymptotic formulas for the implied volatility with four explicit terms were established for the Heston model without jumps in [14] and for the Kou model in [29] and [15]. These formulas can be extended to include five terms and an error estimate. We would also like to bring to the reader's attention the paper [3] concerning the asymptotic behavior of the implied volatility in exponential Lévy models.

We will next briefly overview the contents of the present paper. In subsection 2.1, we define the Mellin convolution and introduce several related notions. Regularly varying functions play 
an important role in the paper. In subsection 2.2, various definitions and facts from the theory of regularly varying functions are gathered, while subsection 2.3 is devoted to Arandelović's theorem and its generalizations (Theorems 8 and 9). In section 3, we introduce the Heston model with double exponential jumps and formulate our main results concerning asymptotic expansions of marginal distribution densities of the stock price process in this model (see Theorems 10-13). In section 4, sharp asymptotic formulas with error estimates are established for the implied volatility in the Heston model with double exponential jumps (see Theorems 15 and 16) and for more general models. In section 5, we study marginal distributions of the exponential Lévy process associated with the perturbed Heston model. The asymptotic behavior of the density of the absolutely continuous part of such a distribution is characterized in Corollary 3. We also complete the proofs of Theorems 10-13 in section 5. Finally, section 6 discusses the implied volatility in the Heston model with jumps distributed according to the symmetric centered NIG law.

2. The Mellin convolution and Arandelović's theorem. In this section, we discuss the Mellin transform and the Mellin convolution, formulate Arandelović theorem concerning the asymptotics of the Mellin convolution, and obtain generalizations of Arandelović's theorem (Theorems 8 and 9).

\subsection{The Mellin transform and the Mellin convolution.}

Definition 1. Let $U$ be a measurable function on $(0, \infty)$. The Mellin transform of $U$ is defined as follows:

$$
M U(z)=\int_{0}^{\infty} t^{-z} U(t) \frac{d t}{t}, \quad z \in \mathbb{C} .
$$

The domain of the Mellin transform of $U$ is the set of all $z \in \mathbb{C}$ for which the integral in (2) converges absolutely.

Definition 2. The Mellin convolution of two real Lebesgue measurable functions $f$ and $g$ on $(0, \infty)$ is defined by

$$
f^{M} \star g(x)=\int_{0}^{\infty} f\left(t^{-1} x\right) g(t) \frac{d t}{t}
$$

for those $x>0$ for which the integral exists.

It is clear that

$$
f^{M} \star g(x)=\int_{0}^{\infty} f\left(t^{-1}\right) g(x t) \frac{d t}{t} .
$$

Moreover,

$$
f^{M} \star g(x)=\widetilde{f} \star \widetilde{g}\left(x^{-1}\right),
$$

where

$$
\widetilde{f}(u)=f\left(u^{-1}\right) \quad \text { and } \quad \widetilde{g}(u)=g\left(u^{-1}\right)
$$

for all $u>0$. We also have

$$
M \widetilde{U}(z)=M U(-z) .
$$


Let $\mu$ be a distribution on $[0, \infty)$, and let $\eta$ be a real number. The moment of order $\eta$ of the distribution $\mu$ is defined as follows:

$$
m_{\eta}(\mu)=\int_{0}^{\infty} t^{\eta} d \mu(t)
$$

It is not hard to see that if $U$ is a distribution density, then $M U(\eta)=m_{-\eta-1}(U)$ for all real numbers $\eta$ in the domain of $M U$.

2.2. Regularly varying functions. In the present subsection, several notions and results from the theory of regularly varying functions are discussed. These functions play an important role in the paper. A rich source of information about regularly varying functions is the book by Bingham, Goldie, and Teugels [9].

Definition 3. A nonnegative measurable function $f$ on $(0, \infty)$ is called regularly varying with index $\rho \in \mathbb{R}$ if for every $\lambda>0$,

$$
\frac{f(\lambda x)}{f(x)} \rightarrow \lambda^{\rho}
$$

as $x \rightarrow \infty$. The class of all regularly varying functions with index $\rho$ is denoted by $R_{\rho}$. Functions from the class $R_{0}$ are called slowly varying functions.

The next result is known as the uniform convergence theorem for regularly varying functions.

Theorem 1 (see Theorem 1.5.2 in [9]). Let $f$ be a nonnegative measurable function on $(0, \infty)$. Then the following are true:

1. Suppose $f \in R_{\rho}$ with $\rho>0$ and $f$ is bounded on every interval $(0, a]$ with $a>0$. Then formula (7) holds uniformly in $\lambda$ on each interval $(0, a], a>0$.

2. The condition $f \in R_{0}$ implies that formula (7) holds uniformly in $\lambda$ on each interval $[a, b]$ with $0<a<b<\infty$.

3. The condition $f \in R_{\rho}$ with $\rho<0$ implies that formula (7) holds uniformly in $\lambda$ on each interval $[b, \infty), b>0$.

Another fundamental result in the theory of slowly varying functions is the representation theorem (see [9, Thm. 1.3.1]).

Theorem 2. For a nonnegative measurable function $l$, the condition $l \in R_{0}$ is equivalent to the

$$
l(x)=c(x) \exp \left\{\int_{a}^{x} \frac{\varepsilon(u)}{u} d u\right\}, \quad x>a,
$$

for some $a>0$, where the functions $c$ and $\varepsilon$ are such that $c(x) \rightarrow c \in(0, \infty)$ as $x \rightarrow \infty$ and $\varepsilon(u) \rightarrow 0$ as $u \rightarrow \infty$.

Definition 4. A function $l \in R_{0}$ is called a normalized slowly varying function provided that the function $x \mapsto c(x)$ in (8) is constant on the interval $[a, \infty)$ for some $a>0$.

Let $l$ be a normalized slowly varying function. Then Theorem 2 shows that

$$
l(x)=\exp \left\{C+\int_{a}^{x} \frac{\varepsilon(u)}{u} d u\right\}, \quad x>a,
$$


for some $C \in \mathbb{R}$ and $a>0$, where the function $\varepsilon$ is such that $\varepsilon(u) \rightarrow 0$ as $u \rightarrow \infty$.

For a normalized slowly varying function $l$, we have

$$
\varepsilon(x)=\frac{x l^{\prime}(x)}{l(x)} \quad \text { almost everywhere }
$$

(see $[9$, p. 15]). If the function $l$ is differentiable, then the equality in (10) holds everywhere on $(a, \infty)$.

Definition 5. A nonnegative measurable function $l$ defined on $(0, \infty)$ belongs to the Zygmund class $\mathcal{Z}$ if, for every $\alpha>0$, the function $\phi_{\alpha}(x)=x^{\alpha} l(x)$ is ultimately increasing and the function $\psi_{\alpha}(x)=x^{-\alpha} l(x)$ is ultimately decreasing.

Remark 1. The class of functions, satisfying the conditions in Definition 5, was introduced by Zygmund (see, e.g., [28, p. 186]), who called such functions slowly varying functions. A generally accepted definition of slow variation is given above (see Definition 3). The term "the Zygmund class" is used in [9, sect. 1.5.3].

The following well-known statement gives a description of the Zygmund class.

Theorem 3 (see Theorem 1.5.5. in [9]). The class $\mathcal{Z}$ coincides with the class of normalized slowly varying functions.

In the present paper, we discuss various asymptotic formulas. Let $\psi_{1}$ and $\psi_{2}$ be real positive functions defined on $(0, \infty)$. Throughout the paper the statement $\phi_{1}(x)=O\left(\phi_{2}(x)\right)$ as $x \rightarrow \infty$ means that there exist $c>0$ and $x_{0}>0$ such that $\left|\phi_{1}(x)\right| \leq c \phi_{2}(x)$ for all $x>x_{0}$, while the statement $\phi_{1}(x) \sim \phi_{2}(x)$ as $x \rightarrow \infty$ means that $\lim _{x \rightarrow \infty} \frac{\phi_{1}(x)}{\phi_{2}(x)}=1$. The explanation of the statements $\phi_{1}(x)=O\left(\phi_{2}(x)\right)$ as $x \rightarrow 0$ and $\phi_{1}(x) \sim \phi_{2}(x)$ as $x \rightarrow 0$ is similar.

The following known definition introduces slowly varying functions with remainder (see [16]; see also [9]).

Definition 6. Let $l$ and $g$ be nonnegative measurable functions on $(0, \infty)$ with $g(x) \rightarrow 0$ as $x \rightarrow \infty$. The function $l$ is called slowly varying with remainder $g$ if for all $\lambda>1$,

$$
\frac{l(\lambda x)}{l(x)}-1=O(g(x))
$$

as $x \rightarrow \infty$.

We will denote the class of slowly varying functions with remainder $g$ by $R_{0}^{g}$. It is not hard to see that $R_{0}^{g} \subset R_{0}$. The uniform convergence theorem for slowly varying functions with remainder is as follows.

Theorem 4 (see Corollary 2.2.1 in [9]). Let $l \in R_{0}^{g}$ where $g \in R_{0}$. Then condition (11) holds uniformly in $\lambda$ on every interval $[1, b], b>1$.

The next statement, which is stronger than Theorem 4, provides a growth estimate in the variable $\lambda$ in the uniform convergence result for slowly varying functions with remainder.

Theorem 5. Fix $\delta \neq 0$, and let $f$ and $g$ be positive functions on $[0, \infty)$ such that $g \in R_{0}$, $g(x) \rightarrow 0$ as $x \rightarrow \infty$, and $f \in R_{0}^{g}$. Suppose also that the functions $f, g, \frac{1}{f}$, and $\frac{1}{g}$ are locally bounded on $[0, \infty)$. Then there exists $A>0$ such that

$$
|f(\lambda x)-f(x)| \leq A f(x) g(x) \max \left\{\lambda^{\delta}, \lambda^{-\delta}\right\}
$$

for all $\lambda>0$ and $x \geq 0$. 
Proof. For $l \in R_{0}$, the class $O \Pi_{l}$ consists of measurable functions $h$ such that for every $\lambda \geq 1, h(\lambda x)-h(x)=O(l(x))$ as $x \rightarrow \infty$ (see estimate (3.0.6) on page 129 in [9]). It follows from the condition $f \in R_{0}^{g}$ that $f \in O \Pi_{f g}$. The previous condition and the local boundedness conditions in the formulation of Theorem 5 allow us to use part (b) of Theorem 3.8.6 in [9]; estimate (12) in Theorem 5 follows from estimate (3.8.10) in [9].

This completes the proof of Theorem 5 .

The structure of slowly varying functions with remainder is known. The next result is the representation theorem for slowly varying functions with remainder (see [16]; see also [9]).

Theorem 6. Let $g \in R_{0}$ and $g(x) \rightarrow 0$ as $x \rightarrow \infty$. Then $l \in R_{0}^{g}$ if and only if

$$
l(x)=\exp \left\{C+O(g(x))+\int_{a}^{x} O(g(t)) t^{-1} d t\right\}
$$

as $x \rightarrow \infty$, where $C \in \mathbb{R}$, and the $O$ functions are locally integrable.

Corollary 1. Let $l \in \mathcal{Z}$. Then $l \in R_{0}^{|\varepsilon|}$, where $\varepsilon$ is the function appearing in formula (9).

Proof. Since $l$ is a normalized slowly varying function, formula (9) holds. This implies that formula (13) holds with $g=|\varepsilon|$. Next, using Theorem 6, we establish Corollary 1.

2.3. Arandelovic's theorem and its generalizations. The next statement was obtained by Arandelović (see [5, 9]).

Theorem 7. Suppose the Mellin transform $M U$ of a measurable function $U$ converges at least in the strip $\sigma \leq \Re(z) \leq \tau$, where $-\infty<\sigma<\tau<\infty$. Let $f$ be a measurable function on $(0, \infty)$, and assume the following two conditions hold:

1. $f(x) \sim x^{\rho} l(x)$ as $x \rightarrow \infty$, where $\rho \in(\sigma, \tau)$ and $l \in R_{0}$.

2. The function $x \mapsto x^{-\sigma} f(x)$ is bounded on every interval $(0, a]$, where $a>0$.

Then

$$
U^{M} \star f(x) \sim M U(\rho)\left[x^{\rho} l(x)\right]
$$

as $x \rightarrow \infty$.

In formula (14), there is no error estimate. The next assertion provides such an estimate under certain additional restrictions.

Theorem 8. Suppose the Mellin transform MU of a measurable function $U$ converges at least in the strip $\sigma \leq \Re(z) \leq \tau$ where $-\infty<\sigma<\tau<\infty$. Let $f$ be a measurable function on $(0, \infty)$, and assume the following conditions hold:

1. $f(x)=x^{\rho} l(x)(1+O(h(x)))$ as $x \rightarrow \infty$ where $\rho \in(\sigma, \tau), l \in R_{0}^{g}$ with $g \in R_{0}$, and $h \in \mathcal{Z}$. The functions $g$ and $h$ in the previous formula satisfy $g(x) \rightarrow 0$ and $h(x) \rightarrow 0$ as $x \rightarrow \infty$.

2. The functions $g, l^{-1}$, and $g^{-1}$ are locally bounded on the interval $\left(x_{0}, \infty\right)$ for some $x_{0}>0$.

3. The function $x \mapsto x^{-\sigma} f(x)$ is bounded on every interval $(0, a]$ where $a>0$. Then

$$
U^{\stackrel{M}{\star}} f(x)=M U(\rho)\left[x^{\rho} l(x)\right](1+O(g(x))+O(h(x)))
$$

as $x \rightarrow \infty$. 
Proof. Let us first assume that

$$
f(y)=y^{\rho} l(y) \quad \text { for } \quad y>y_{0}
$$

and then put $x_{1}=\max \left\{x_{0}, y_{0}\right\}$, where $x_{0}$ is as in condition 2 . Then we have

$$
\begin{aligned}
U \stackrel{M}{\star} f(x) & =\int_{0}^{\frac{x_{1}}{x}} f(x v) U\left(v^{-1}\right) \frac{d v}{v}+x^{\rho} \int_{\frac{x_{1}}{x}}^{\infty} v^{\rho} l(x v) U\left(v^{-1}\right) \frac{d v}{v} \\
& =I_{1}(x)+I_{2}(x) .
\end{aligned}
$$

Since $x v<x_{1}$ in the first integral in (17), condition 3 implies the following:

$$
\begin{aligned}
I_{1}(x) & \leq c x^{\sigma} \int_{0}^{\frac{x_{1}}{x}} v^{\sigma} U\left(v^{-1}\right) \frac{d v}{v}=c x^{\sigma} \int_{\frac{x}{x_{1}}}^{\infty} y^{-\sigma-1} U(y) d y \\
& \leq c M U(\sigma) x^{\sigma} .
\end{aligned}
$$

Fix $\varepsilon>0$ such that $\varepsilon<\rho-\sigma$ and take into account that $l g \in R_{0}$. Then the previous estimates imply that $I_{1}(x)=O\left(x^{\sigma}\right)=O\left(x^{\rho-\varepsilon}\right)$, and hence

$$
I_{1}(x)=O\left(x^{\rho} l(x) g(x)\right) \quad \text { as } \quad x \rightarrow \infty .
$$

It follows that the integral $I_{1}$ can be incorporated into the error term in formula (15).

Our next goal is to estimate the integral $I_{2}$. It is clear that

$$
\begin{aligned}
I_{2}(x)= & x^{\rho} \int_{\frac{x_{1}}{x}}^{\infty}[l(x v)-l(x)] v^{\rho} U\left(v^{-1}\right) \frac{d v}{v}+x^{\rho} l(x) \int_{\frac{x_{1}}{x}}^{\infty} v^{\rho} U\left(v^{-1}\right) \frac{d v}{v} \\
= & x^{\rho} l(x) \int_{0}^{\infty} v^{\rho} U\left(v^{-1}\right) \frac{d v}{v}-x^{\rho} l(x) \int_{0}^{\frac{x_{1}}{x}} v^{\rho} U\left(v^{-1}\right) \frac{d v}{v} \\
& +x^{\rho} \int_{\frac{x_{1}}{x}}^{\infty}[l(x v)-l(x)] v^{\rho} U\left(v^{-1}\right) \frac{d v}{v} \\
= & M U(\rho)\left[x^{\rho} l(x)\right]-x^{\rho} l(x) \int_{\frac{x}{x_{1}}}^{\infty} y^{-\rho} U(y) \frac{d y}{y} \\
& +x^{\rho} \int_{\frac{x_{1}}{x}}^{\infty}[l(x v)-l(x)] v^{\rho} U\left(v^{-1}\right) \frac{d v}{v} \\
= & M U(\rho)\left[x^{\rho} l(x)\right]+J_{1}(x)+J_{2}(x) .
\end{aligned}
$$

Fix $\varepsilon>0$ such that $\varepsilon<\rho-\sigma$. Then, for large values of $x$, we have

$$
\left|J_{1}(x)\right| \leq x^{\rho} l(x) \int_{\frac{x}{x_{1}}}^{\infty} y^{-\sigma-\varepsilon} U(y) \frac{d y}{y} \leq x^{\rho-\varepsilon} l(x) x_{1}^{\varepsilon} M U(\sigma) .
$$

Since $g \in R_{0}$ and $M U(\sigma)<\infty$, we obtain

$$
J_{1}(x)=O\left(x^{\rho} l(x) g(x)\right) \quad \text { as } \quad x \rightarrow \infty .
$$


It remains to estimate $J_{2}$. Denote by $\widetilde{l}$ and $\widetilde{g}$ the functions $l$ and $g$ extrapolated by positive constants from $\left(x_{1}, \infty\right)$ to $[0, \infty)$. Then $\widetilde{l} \in R_{0}^{\widetilde{g}}$. Moreover, condition 2 and the definition of the functions $\widetilde{l}$ and $\widetilde{g}$ imply that the functions $\widetilde{l}, \widetilde{g}, \widetilde{l}^{-1}$, and $\widetilde{g}^{-1}$ are locally bounded on $[0, \infty)$. Now, using Theorem 5 , we see that for every $\delta>0$ there exists a constant $A>0$ such that

$$
|\widetilde{l}(x v)-\widetilde{l}(x)| \leq A \widetilde{l}(x) \widetilde{g}(x) \max \left\{v^{\delta}, v^{-\delta}\right\}
$$

for all $v>0$ and $x \geq 0$. Recalling the definition of the functions $\widetilde{l}$ and $\widetilde{g}$, we see that for $x>x_{1}$ and $v>\frac{x_{1}}{x}$

$$
|l(x v)-l(x)| \leq A l(x) g(x) \max \left\{v^{\delta}, v^{-\delta}\right\} .
$$

It follows from the estimate in (21) that for every $\delta>0$ there exists $x_{1}>0$ depending on $\delta$ and such that

$$
\begin{aligned}
\left|J_{2}(x)\right| & \leq A x^{\rho} l(x) g(x) \int_{\frac{x_{1}}{x}}^{\infty} \max \left\{v^{\delta}, v^{-\delta}\right\} v^{\rho} U\left(v^{-1}\right) \frac{d v}{v} \\
& \leq A x^{\rho} l(x) g(x) \int_{0}^{\infty} \max \left\{v^{\delta}, v^{-\delta}\right\} v^{\rho} U\left(v^{-1}\right) \frac{d v}{v}
\end{aligned}
$$

for all $x>x_{1}$. It is not hard to see that for small enough values of $\delta$, the last integral in (22) is finite. Here we use the fact that $M U(s)<\infty$ for all $\sigma \leq s \leq \tau$ and the inequalities $\sigma<\rho<\tau$. Now, (22) implies that

$$
J_{2}(x)=O\left(x^{\rho} l(x) g(x)\right) \quad \text { as } \quad x \rightarrow \infty .
$$

Next, taking into account formulas (19), (20), and (23), we obtain

$$
I_{2}(x)=M U(\rho)\left[x^{\rho} l(x)\right]+O\left(x^{\rho} l(x) g(x)\right)
$$

as $x \rightarrow \infty$. Finally, it is easy to see that formulas (17), (18), and (24) imply formula (15). This establishes Theorem 8 in a special case where (16) holds.

We will next prove Theorem 8 in the general case. Suppose the conditions in the formulation of Theorem 8 hold. Then there exists $x_{1}>0$ such that $f(x)=x^{\rho} l(x)+x^{\rho} l(x) \eta(x)$ for all $x>x_{1}$, where $\eta$ is a measurable function such that $|\eta(x)| \leq A h(x)$ for all $x>x_{1}$ and for some constant $A>0$. Put $f_{1}(x)=f(x) \chi_{\left\{0<x<x_{1}\right\}}, f_{2}(x)=x^{\rho} l(x) \chi_{\left\{x>x_{1}\right\}}$, and $f_{3}(x)=x^{\rho} l(x) \eta(x) \chi_{\left\{x>x_{1}\right\}}$. Then

$$
U^{M} \star f^{\prime}(x)=U^{\stackrel{M}{\star}} f_{1}(x)+U^{\stackrel{M}{\star}} f_{2}(x)+U^{\stackrel{M}{\star}} f_{3}(x) .
$$

Applying the special case of Theorem 8 established above to the function $f_{2}$, we obtain

$$
U^{M} \star f_{2}(x)=M U(\rho)\left[x^{\rho} l(x)\right](1+O(g(x)))
$$

as $x \rightarrow \infty$. In addition, reasoning as in the proof of (18), we obtain

$$
U^{M} \star f_{1}(x)=O\left(x^{\rho} l(x) g(x)\right)
$$


as $x \rightarrow \infty$.

We will next estimate the function $U^{M} f_{3}$, using the same ideas as in the estimate for the function $U \stackrel{M}{\star} f_{2}$. However, we will use the function $\widetilde{l}=l h$ instead of the function $l$. Recall that $l \in R_{0}^{g}$. Moreover, $h \in R_{0}^{|\varepsilon|}$, where $\varepsilon$ is the function appearing in formula (9) for the function $h$ (see Corollary 1 ).

We have

$$
\left|\frac{\widetilde{l}(x v)}{\widetilde{l}(x)}-1\right| \leq\left|\frac{l(x v)}{l(x)}-1\right| \frac{h(x v)}{h(x)}+\left|\frac{h(x v)}{h(x)}-1\right| .
$$

Therefore, $\tilde{l} \in R_{0}^{g+|\varepsilon|}$. It is clear that

$$
\left|U^{M} f_{3}(x)\right| \leq A x^{\rho} \int_{\frac{x_{1}}{x}}^{\infty} v^{\rho} l(x v) h(x v) U\left(v^{-1}\right) \frac{d v}{v} .
$$

Now, reasoning as in the proof of formula (24), we see that

$$
\begin{aligned}
U^{M} \star f_{3}(x) & =O\left(x^{\rho} l(x)[h(x)+h(x) g(x)+h(x)|\varepsilon(x)|]\right) \\
& =O\left(x^{\rho} l(x) h(x)\right)
\end{aligned}
$$

as $x \rightarrow \infty$.

Finally, taking into account formulas (25)-(28), we see that formula (15) holds. This completes the proof of Theorem 8 .

A similar theorem characterizes the asymptotic behavior of the Mellin convolution near zero.

Theorem 9. Suppose the Mellin transform MU of a measurable function $U$ converges at least in the strip $\sigma \leq \Re(z) \leq \tau$, where $-\infty<\sigma<\tau<\infty$. Let $f$ be a measurable function on $(0, \infty)$, and assume the following conditions hold:

1. $f\left(y^{-1}\right)=y^{-\rho} l(y)(1+O(h(y)))$ as $y \rightarrow \infty$, where $\rho \in(\sigma, \tau), l \in R_{0}^{g}$ with $g \in R_{0}$, and $h \in Z$. The functions $g$ and $h$ in the previous formula satisfy $g(y) \rightarrow 0$ and $h(y) \rightarrow 0$ as $y \rightarrow \infty$.

2. The functions $g, l^{-1}$, and $g^{-1}$ are locally bounded on the interval $\left(x_{0}, \infty\right)$ for some $x_{0}>0$.

3. The function $y \mapsto y^{\tau} f\left(y^{-1}\right)$ is bounded on every interval $(0, a]$, where $a>0$. Then

$$
U^{M} \star f(x)=M U(\rho)\left[x^{\rho} l\left(x^{-1}\right)\right]\left(1+O\left(g\left(x^{-1}\right)\right)+O\left(h\left(x^{-1}\right)\right)\right)
$$

as $x \rightarrow 0$.

Proof. Theorem 9 follows from Theorem 8, applied to the functions $\widetilde{f}(x)=f\left(x^{-1}\right)$ and $\widetilde{U}(x)=U\left(x^{-1}\right)$. Here we take into account (3), (4), and (5).

3. The Heston model with double exponential jumps. Main results. In this section, we gather several known results for the Heston model, which is a popular stochastic volatility model. The stock price process $X$ and the variance process $Y$ in the Heston model satisfy the following system of stochastic differential equations:

$$
\left\{\begin{array}{l}
d X_{t}=\mu X_{t} d t+\sqrt{Y_{t}} X_{t} d W_{t} \\
d Y_{t}=\left(a-b Y_{t}\right) d t+c \sqrt{Y_{t}} d Z_{t}
\end{array}\right.
$$


where $\mu \in \mathbb{R}, a \geq 0, b \geq 0, c>0$. In (29), $W$ and $Z$ are correlated standard Brownian motions such that $d\langle W, Z\rangle_{t}=\rho d t$ with $\rho \in(-1,1)$. It will be assumed in what follows that the interest rate $r$ is equal to zero. In the Heston model, the distribution $\mu_{t}$ of the stock price $X_{t}$ admits density $D_{t}^{(1)}$. The initial conditions for the processes $X$ and $Y$ will be denoted by $x_{0}$ and $y_{0}$, respectively. The Heston model was introduced and studied in [20].

We have

$$
X_{t}=x_{0} \exp \left\{\mu t-\frac{1}{2} \int_{0}^{t} Y_{s} d s+\int_{0}^{t} \sqrt{Y_{s}} d W_{s}\right\}
$$

and the following formulas hold for the density $D_{t}^{(1)}$ in the Heston model in the case where $\mu=0$ and $x_{0}=1$ :

$$
D_{t}^{(1)}(x)=A_{1} x^{-A_{3}} \exp \left\{A_{2} \sqrt{\log x}\right\}(\log x)^{-\frac{3}{4}+\frac{a}{c^{2}}}\left(1+O\left((\log x)^{-\frac{1}{2}}\right)\right)
$$

as $x \rightarrow \infty$, and

$$
D_{t}^{(1)}(x)=\widetilde{A}_{1} x^{\widetilde{A}_{3}} \exp \left\{\widetilde{A}_{2} \sqrt{\log \frac{1}{x}}\right\}\left(\log \frac{1}{x}\right)^{-\frac{3}{4}+\frac{a}{c^{2}}}\left(1+O\left(\left(\log \frac{1}{x}\right)^{-\frac{1}{2}}\right)\right)
$$

as $x \rightarrow 0$. The constants appearing in formulas (30) and (31) will be described below (see Remark 4). Formulas (30) and (31) were obtained in [18] in the case where $\rho=0$ and in [13] for $-1<\rho<0$. A more detailed discussion of those and similar results can be found in [17].

For general $x_{0}$ and $\mu$, we have

$$
D_{t}^{(1)}(x)=B_{1} x^{-A_{3}} \exp \left\{A_{2} \sqrt{\log x}\right\}(\log x)^{-\frac{3}{4}+\frac{a}{c^{2}}}\left(1+O\left((\log x)^{-\frac{1}{2}}\right)\right)
$$

as $x \rightarrow \infty$, and

$$
D_{t}^{(1)}(x)=\widetilde{B}_{1} x^{\widetilde{A}_{3}} \exp \left\{\widetilde{A}_{2} \sqrt{\log \frac{1}{x}}\right\}\left(\log \frac{1}{x}\right)^{-\frac{3}{4}+\frac{a}{c^{2}}}\left(1+O\left(\left(\log \frac{1}{x}\right)^{-\frac{1}{2}}\right)\right)
$$

as $x \rightarrow 0$. In (32) and (33), the constants $B_{1}$ and $\widetilde{B}_{1}$ are defined as follows:

$$
B_{1}=A_{1}\left(x_{0} e^{\mu t}\right)^{-A_{3}-1}
$$

and

$$
\widetilde{B}_{1}=\widetilde{A}_{1}\left(x_{0} e^{\mu t}\right)^{\widetilde{A}_{3}-1}
$$

The proof of (33) uses (30) and the following simple formulas:

$$
\exp \left\{r_{1} \sqrt{\log x+r_{2}}\right\}=\exp \left\{r_{1} \sqrt{\log x}\right\}\left(1+O\left((\log x)^{-\frac{1}{2}}\right)\right), \quad x \rightarrow \infty, \quad r_{1} \in \mathbb{R}, \quad r_{2} \in \mathbb{R},
$$

and

$$
\left(\log x+r_{3}\right)^{r_{4}}=(\log x)^{r_{4}}\left(1+O\left((\log x)^{-\frac{1}{2}}\right)\right), \quad x \rightarrow \infty, \quad r_{3} \in \mathbb{R}, \quad r_{4} \in \mathbb{R} .
$$

The proof of (33) is similar. It is based on formula (31). 
Let $N$ be a standard Poisson process with intensity $\lambda>0$, and consider a compound Poisson process defined by

$$
J_{t}=\sum_{i=1}^{N_{t}}\left(V_{i}-1\right), \quad t \geq 0,
$$

where $V_{i}$ are positive identically distributed random variables such that the distribution density $g$ of the random variable $U_{i}=\log V_{i}$ is double exponential. This means that

$$
g(u)=p \eta_{1} e^{-\eta_{1} u} \chi_{\{u \geq 0\}}+q \eta_{2} e^{\eta_{2} u} \chi_{\{u<0\}},
$$

where $\eta_{1}>1, \eta_{2}>0$, and $p$ and $q$ are positive numbers such that $p+q=1$. The condition $\eta_{1}>1$ is necessary and sufficient for the random variable $J_{t}$ to have finite expectation.

S. Kou introduced and studied a perturbation of the Black-Scholes model based on the jump process described above (see [22]; see also [23]). In [19], we considered a similar perturbation of the Heston model. The stock price process and the variance process $Y$ in the perturbed Heston model satisfy the following system of stochastic differential equations:

$$
\left\{\begin{array}{l}
d \widetilde{X}_{t}=\mu \widetilde{X}_{t-} d t+\sqrt{Y_{t}} \widetilde{X}_{t-} d W_{t}+\widetilde{X}_{t-} d J_{t} \\
d Y_{t}=\left(a-b Y_{t}\right) d t+c \sqrt{Y_{t}} d Z_{t}
\end{array}\right.
$$

It is assumed in (34) that the compound Poisson process $J$ is independent of the standard Brownian motions $W$ and $Z$. The initial conditions for the processes $\widetilde{X}$ and $Y$ will be denoted by $x_{0}$ and $y_{0}$, respectively.

It is not hard to see that

$$
\widetilde{X}_{t}=x_{0} \exp \left\{\mu t-\frac{1}{2} \int_{0}^{t} Y_{s} d s+\int_{0}^{t} \sqrt{Y_{s}} d W_{s}+\sum_{i=1}^{N_{t}} U_{i}\right\} .
$$

The validity of the equality in (35) follows from the Doléans-Dade formula (see, for example, [25]).

The Heston model with double exponential jumps is a mixed stochastic model. Indeed, using formula (35), we can split the process $\widetilde{X}$ into the product of the following processes:

$$
X_{t}^{(1)}=x_{0} \exp \left\{\mu t-\frac{1}{2} \int_{0}^{t} Y_{s} d s+\int_{0}^{t} \sqrt{Y_{s}} d W_{s}\right\}
$$

and

$$
X_{t}^{(2)}=\exp \left\{\sum_{i=1}^{N_{t}} U_{i}\right\}
$$

We have $X^{(1)}=X$, where $X$ is the stock price process in the Heston model described by (29), and for every $t \geq 0$, the following inequality holds: $\mathbb{E}\left[X_{t}^{(2)}\right]<\infty$ (see Remark 8 below). 
We will next formulate our main results concerning the asymptotic behavior of the stock price density in the Heston model with double exponential jumps. We first consider the case of the perturbed Heston model where the jump part dominates.

Theorem 10. Fix $t>0$, and suppose $1+\eta_{1}<A_{3}$. Then the following asymptotic formula holds for the stock price density $D_{t}$ in the Heston model with double exponential jumps:

$$
\begin{aligned}
D_{t}(x)= & \frac{1}{2 \sqrt{\pi}} m_{\eta_{1}}\left(D_{t}^{(1)}\right)\left(\eta_{1} \lambda t p\right)^{\frac{1}{4}} \exp \left\{\frac{\eta_{2} \lambda t q}{\eta_{1}+\eta_{2}}-\lambda t\right\}(\log x)^{-\frac{3}{4}} \\
& \times \exp \left\{2 \sqrt{\eta_{1} \lambda t p} \sqrt{\log x}\right\} x^{-\eta_{1}-1}\left(1+O\left((\log x)^{-\frac{1}{2}}\right)\right)
\end{aligned}
$$

as $x \rightarrow \infty$. Here $D_{t}^{(1)}$ is the density of the random variable $X_{t}^{(1)}$ defined by (36).

Theorem 11. Fix $t>0$, and suppose $\widetilde{A}_{3}>\eta_{2}-1$. Then the following asymptotic formula holds for the stock price density $D_{t}$ in the Heston model with double exponential jumps:

$$
\begin{aligned}
D_{t}(x)= & \frac{1}{2 \sqrt{\pi}} m_{-\eta_{2}}\left(D_{t}^{(1)}\right)\left(\eta_{2} \lambda t q\right)^{\frac{1}{4}} \exp \left\{\frac{\eta_{1} \lambda t p}{\eta_{1}+\eta_{2}}-\lambda t\right\}\left(\log \frac{1}{x}\right)^{-\frac{3}{4}} \\
& \times \exp \left\{2 \sqrt{\eta_{2} \lambda t q} \sqrt{\log \frac{1}{x}}\right\} x^{\eta_{2}-1}\left(1+O\left(\left(\log \frac{1}{x}\right)^{-\frac{1}{2}}\right)\right)
\end{aligned}
$$

as $x \rightarrow 0$.

Remark 2. The symbols $m_{\eta_{1}}\left(D_{t}^{(1)}\right)$ and $m_{-\eta_{2}}\left(D_{t}^{(1)}\right)$ in formulas (38) and (39) stand for the moments of the marginal density $D_{t}^{(1)}$ (see (6)). Formula (38) becomes meaningless if $1+\eta_{1}=A_{3}$. Indeed, if the previous equality holds, then $m_{\eta_{1}}\left(D_{t}^{(1)}\right)=\infty$ (use (30)). Similarly, if $\widetilde{A}_{3}=\eta_{2}-1$, then $m_{-\eta_{2}}\left(D_{t}^{(1)}\right)=\infty$ (use (31)), and formula (39) does not hold.

We will next explain how the density $D_{t}$ behaves in the case where the Heston part dominates.

Theorem 12. Fix $t>0$, and suppose $1+\eta_{1}>A_{3}$. Then the following asymptotic formula holds for the stock price density $D_{t}$ in the Heston model with double exponential jumps:

$$
\begin{aligned}
D_{t}(x)= & {\left[e^{-\lambda t}+m_{A_{3}-1}(H(t, \cdot))\right] B_{1} x^{-A_{3}} \exp \left\{A_{2} \sqrt{\log x}\right\}(\log x)^{-\frac{3}{4}+\frac{a}{c^{2}}} } \\
& \times\left(1+O\left((\log x)^{-\frac{1}{2}}\right)\right)
\end{aligned}
$$

as $x \rightarrow \infty$.

Theorem 13. Fix $t>0$, and suppose $\widetilde{A}_{3}<\eta_{2}-1$. Then the following asymptotic formula holds for the stock price density $D_{t}$ in the Heston model with double exponential jumps:

$$
\begin{aligned}
D_{t}(x)= & {\left[e^{-\lambda t}+m_{-\widetilde{A}_{3}-1}(H(t, \cdot))\right] \widetilde{B}_{1} x^{\widetilde{A}_{3}} \exp \left\{\widetilde{A}_{2} \sqrt{\log \frac{1}{x}}\right\}\left(\log \frac{1}{x}\right)^{-\frac{3}{4}+\frac{a}{c^{2}}} } \\
& \times\left(1+O\left(\left(\log \frac{1}{x}\right)^{-\frac{1}{2}}\right)\right)
\end{aligned}
$$

as $x \rightarrow 0$. 
Remark 3. Recall that the symbols $m_{A_{3}-1}\left(\mu_{t}^{(2)}\right)$ and $m_{-\widetilde{A}_{3-1}}\left(\mu_{t}^{(2)}\right)$ in formulas (40) and (41) stand for the moments of the marginal distribution $\mu_{t}^{(2)}$ (see (6)). In the extreme case where $1+\eta_{1}=A_{3}$, formula (40) does not hold, since under the previous condition, we have $m_{-\widetilde{A}_{3}-1}\left(\mu_{t}^{(2)}\right)=\infty$ (use (57), (58), and (95)). Similarly, formula (41) is not valid if $\widetilde{A}_{3}=\eta_{2}-1$, since in this case we have $m_{-\widetilde{A}_{3}-1}\left(\mu_{t}^{(2)}\right)=\infty$ (use (57), (58), and (96)).

Remark 4. In this remark, we provide explicit formulas for the constants appearing in formulas (30) and (31) (see $[13,17]$ ). Given $s \geq 1$, define the explosion time for the moment of order $s$ by

$$
T^{*}(s)=\sup \left\{t \geq 0: \mathbb{E}\left[X_{t}^{s}\right]<\infty\right\},
$$

and for any $t>0$, let the $s_{+}$be the upper critical moment defined by

$$
s_{+}=s_{+}(t)=\sup \left\{s \geq 1: \mathbb{E}\left[X_{t}^{s}\right]<\infty\right\} .
$$

For the Heston model, the explosion time $T^{*}$ is explicitly known (see [4, 21]). The critical moment, for fixed $t$, can then be determined from $T^{*}\left(s_{+}(t)\right)=t$. The previous equality shows that $s_{+}(t) \geq 1$ is the generalized inverse of the function $T^{*}(\cdot)$.

The lower critical moment is defined as follows:

$$
s_{-}=s_{-}(t)=\inf \left\{s \leq 0: \mathbb{E}\left[X_{t}^{s}\right]<\infty\right\} .
$$

For fixed $t>0$, the quantities

$$
\sigma_{+}=-\left.\frac{\partial T^{*}(s)}{\partial s}\right|_{s=s_{+}} \quad \text { and } \quad \kappa_{+}=\left.\frac{\partial^{2} T^{*}(s)}{\partial s^{2}}\right|_{s=s_{+}}
$$

are called the upper critical slope and the upper critical curvature, respectively. Similarly, the lower critical slope and curvature are defined by

$$
\sigma_{-}=-\left.\frac{\partial T^{*}(s)}{\partial s}\right|_{s=s_{-}} \quad \text { and } \quad \kappa_{-}=\left.\frac{\partial^{2} T^{*}(s)}{\partial s^{2}}\right|_{s=s_{-}},
$$

respectively. In formula (30), the constants $A_{1}, A_{2}$, and $A_{3}$ are given by

$$
\begin{gathered}
A_{1}=\frac{1}{\sqrt{\pi}} 2^{-\frac{3}{4}-\frac{a}{c^{2}}} y_{0}^{\frac{1}{4}-\frac{a}{c^{2}}} c^{\frac{2 a}{c^{2}}-\frac{1}{2}} \sigma_{+}^{-\frac{a}{c^{2}}-\frac{1}{4}} \\
\times \exp \left\{-y_{0}\left(\frac{c \rho s_{+}-b}{c^{2}}+\frac{\kappa_{+}}{c^{2} \sigma_{+}^{2}}\right)-\frac{a t}{c^{2}}\left(c \rho s_{+}-b\right)\right\} \\
\times\left\{\frac{2 \sqrt{\left(b-c \rho s_{+}\right)^{2}+c^{2}\left(s_{+}-s_{+}^{2}\right)}}{c^{2} s_{+}\left(s_{+}-1\right) \sinh \left[\frac{t}{2} \sqrt{\left(b-c \rho s_{+}\right)^{2}+c^{2}\left(s_{+}-s_{+}^{2}\right)}\right]},\right. \\
A_{2}=2 \sqrt{2 y_{0}} c^{-1} \sigma_{+}^{-\frac{1}{2}}, \quad A_{3}=s_{+}+1 .
\end{gathered}
$$


In addition, the constants $\widetilde{A}_{1}, \widetilde{A}_{2}$, and $\widetilde{A}_{3}$ in formula (31) are as follows:

$$
\begin{aligned}
& \widetilde{A}_{1}=\frac{1}{2 \sqrt{\pi}}\left(2 y_{0}\right)^{1 / 4-a / c^{2}} c^{2 a / c^{2}-1 / 2} \sigma_{-}^{-a / c^{2}-1 / 4} \\
& \times \exp \left\{-y_{0}\left(\frac{s_{-} \rho c-b}{c^{2}}+\frac{\kappa_{-}}{c^{2} \sigma_{-}^{2}}\right)-\frac{a t}{c^{2}}\left(c \rho s_{-}-b\right)\right\} \\
& \times\left(\frac{2 \sqrt{b^{2}-2 b c \rho s_{-}+c^{2} s_{-}\left(1-\left(1-\rho^{2}\right) s_{-}\right)}}{c^{2} s_{-}\left(s_{-}-1\right) \sinh \frac{t}{2} \sqrt{b^{2}-2 b c \rho s_{-}+c^{2} s_{-}\left(1-\left(1-\rho^{2}\right) s_{-}\right)}}\right)^{\frac{2 a}{c^{2}}}, \\
& \widetilde{A}_{2}=2 \sqrt{2 y_{0}} c^{-1} \sigma_{-}^{-\frac{1}{2}}, \quad \widetilde{A}_{3}=-\left(s_{-}+1\right) .
\end{aligned}
$$

The constants described above depend on the parameter $t$.

Remark 5. It follows from (30) and (31) that the interval $\left(-A_{3}, \widetilde{A}_{3}\right)$ belongs to the domain of the Mellin transform $M D_{t}^{(1)}$.

We will prove Theorems 10-13 in section 5 .

4. Smile asymptotics in the Heston model with double exponential jumps. In order to create a risk-neutral environment, we assume that the following no-arbitrage condition holds for the parameters in the perturbed Heston model:

$$
\mu=\lambda\left(\frac{q}{\eta_{2}+1}-\frac{p}{\eta_{1}-1}\right)
$$

Here we take into account that $r=0$. Then the process $\tilde{X}$ defined by (35) is a martingale (see [17, sect. 10.8]). The proof uses the mean-correcting argument (see, e.g., Lemma 10.40 in [17] or [26, pp. 79-80]). It will be assumed in the present section that condition (42) holds.

The call and put pricing functions $C$ and $P$ in the Heston model with double exponential jumps are defined by $C(T, K)=\mathbb{E}\left[\left(\widetilde{X}_{T}-K\right)^{+}\right]$and $P(T, K)=\mathbb{E}\left[\left(K-\widetilde{X}_{T}\right)^{+}\right]$, respectively. In the previous formulas, $T$ is the maturity and $K$ is the strike price. The implied volatility $I(T, K), T>0, K>0$, in the Heston model with double exponential jumps is defined as follows. Given $T$ and $K$, the implied volatility $I(T, K)$ is equal to the value of the volatility $\sigma=\sigma(T, K)$ in the Black-Scholes model such that $C(T, K)=C_{B S}(T, K, \sigma)$. Here the symbol $C_{B S}$ stands for the call pricing function in the Black-Scholes model. In what follows, the maturity $T$ will be fixed, and we will consider the functions $C, P$, and $I$ as functions of only the strike price $K$.

The asymptotic behavior of the implied volatility $I$ in the Heston model with double exponential jumps will be characterized utilizing the asymptotic formulas for the stock price densities provided in Theorems 10-13. We will start with the case of large strikes. Analyzing the formulas in Theorems 10 and 12, we see that it is important to understand how the implied volatility behaves if the stock price density $D_{T}$ satisfies the condition

$$
D_{T}(x)=r_{1} x^{-r_{3}} \exp \left\{r_{2} \sqrt{\log x}\right\}(\log x)^{r_{4}}\left(1+O\left((\log x)^{-\frac{1}{2}}\right)\right)
$$

as $x \rightarrow \infty$, where $r_{1}>0, r_{2} \geq 0, r_{3}>2$, and $r_{4} \in \mathbb{R}$. 
Theorem 14. Suppose condition (43) holds. Then the following asymptotic formula is valid for the implied volatility:

$$
\begin{aligned}
& I(K)=\frac{\sqrt{2}}{\sqrt{T}}\left(\sqrt{r_{3}-1}-\sqrt{r_{3}-2}\right) \sqrt{\log \frac{K}{x_{0}}}+\frac{r_{2}}{\sqrt{2 T}}\left(\frac{1}{\sqrt{r_{3}-2}}-\frac{1}{\sqrt{r_{3}-1}}\right) \\
& +\frac{2 r_{4}+1}{2 \sqrt{2 T}}\left(\frac{1}{\sqrt{r_{3}-2}}-\frac{1}{\sqrt{r_{3}-1}}\right) \frac{\log \log \frac{K}{x_{0}}}{\sqrt{\log \frac{K}{x_{0}}}} \\
& \quad+\left[\frac{1}{\sqrt{2 T}} \log \frac{\sqrt{r_{3}-1} \sqrt{r_{3}-2}\left(\sqrt{r_{3}-1}-\sqrt{r_{3}-2}\right)}{2 \sqrt{\pi} r_{1}}+\frac{r_{2}^{2}}{4 \sqrt{2 T}}\left(\frac{1}{\left(r_{3}-2\right)^{\frac{3}{2}}}-\frac{1}{\left(r_{3}-1\right)^{\frac{3}{2}}}\right)\right] \frac{1}{\sqrt{\log \frac{K}{x_{0}}}}
\end{aligned}
$$

$$
+\frac{r_{2}\left(2 r_{4}+1\right)}{4 \sqrt{2 T}}\left(\frac{1}{\left(r_{3}-2\right)^{\frac{3}{2}}}-\frac{1}{\left(r_{3}-1\right)^{\frac{3}{2}}}\right) \frac{\log \log \frac{K}{x_{0}}}{\log \frac{K}{x_{0}}}+O\left(\frac{1}{\log \frac{K}{x_{0}}}\right)
$$

as $K \rightarrow \infty$.

Proof. For the sake of simplicity, we assume $x_{0}=1$. The proof in the general case is similar.

It follows from (43), Corollary 7.13 in [17], and Theorem 8.10 in [17] that as $K \rightarrow \infty$,

$$
C(K)=\frac{r_{1}}{\left(r_{3}-1\right)\left(r_{3}-2\right)}(\log K)^{r_{4}} \exp \left\{r_{2} \sqrt{\log K}\right\} K^{2-r_{3}}\left(1+O\left(\frac{1}{\sqrt{\log K}}\right)\right) .
$$

Therefore, as $K \rightarrow \infty$,

$$
\begin{aligned}
\log \frac{1}{C(K)}= & \log \frac{\left(r_{3}-1\right)\left(r_{3}-2\right)}{r_{1}}-r_{4} \log \log K-r_{2} \sqrt{\log K}+\left(r_{3}-2\right) \log K \\
& +O\left(\frac{1}{\sqrt{\log K}}\right) .
\end{aligned}
$$

Moreover, the mean value theorem and (46) imply that

$$
\log \log \frac{1}{C(K)}=\log \log K+\log \left(A_{3}-2\right)+O\left(\frac{1}{\sqrt{\log K}}\right)
$$

as $K \rightarrow \infty$. Next, using Theorem 9.16 in [17] with $\lambda=r_{3}-2$ and $\Lambda(K)=\sqrt{\log K}$, we get

$$
I(K)=\frac{\sqrt{2}}{\sqrt{T}}\left[\sqrt{\left(r_{3}-1\right) \log K+L(K)}-\sqrt{\left(r_{3}-2\right) \log K+L(K)}\right]+O\left(\frac{1}{\log K}\right)
$$

as $K \rightarrow \infty$, where

$$
\begin{aligned}
L(K)= & -r_{2} \sqrt{\log K}-\left(r_{4}+\frac{1}{2}\right) \log \log K+\log \frac{\left(r_{3}-1\right)\left(r_{3}-2\right)}{r_{1}} \\
& -\log \left(r_{3}-2\right)+\log \frac{\sqrt{r_{3}-1}-\sqrt{r_{3}-2}}{2 \sqrt{\pi} r_{3}-1} .
\end{aligned}
$$


It follows from (47) that

$$
\begin{aligned}
& I(K)= \frac{\sqrt{2}}{\sqrt{T}}\left[\sqrt{r_{3}-1} \sqrt{\log K} \sqrt{1+\frac{L(K)}{\left(r_{3}-1\right) \log K}}-\sqrt{r_{3}-2} \sqrt{\log K} \sqrt{1+\frac{L(K)}{\left(r_{3}-2\right) \log K}}\right] \\
&(48) \quad+O\left(\frac{1}{\log K}\right)
\end{aligned}
$$

as $K \rightarrow \infty$. Now, using the formula $\sqrt{1+h}=1+\frac{1}{2} h-\frac{1}{8} h^{2}+O\left(h^{3}\right), h \rightarrow 0$, in (48), and making simplifications, we obtain formula (44) with $x_{0}=1$.

This completes the proof of Theorem 14.

The next theorem characterizes the asymptotic behavior of the implied volatility at large strikes in the Heston model with double exponential jumps.

Theorem 15. Let $T>0$, and suppose $1+\eta_{1}<A_{3}$. Then formula (44) holds with

$$
\begin{gathered}
r_{1}=\frac{1}{2 \sqrt{\pi}} m_{\eta_{1}}\left(D_{T}^{(1)}\right)\left(\eta_{1} \lambda T p\right)^{\frac{1}{4}} \exp \left\{\frac{\eta_{2} \lambda T q}{\eta_{1}+\eta_{2}}-\lambda T\right\}, \\
r_{2}=2 \sqrt{\eta_{1} \lambda T p}, \quad r_{3}=\eta_{1}+1, \quad \text { and } \quad r_{4}=-\frac{3}{4} .
\end{gathered}
$$

On the other hand, if $1+\eta_{1}>A_{3}$, then formula (44) holds with

$$
\begin{gathered}
r_{1}=\left[e^{-\lambda T}+m_{A_{3}-1}(H(T, \cdot))\right] B_{1}, \\
r_{2}=A_{2}, \quad r_{3}=A_{3}, \quad \text { and } \quad r_{4}=-\frac{3}{4}+\frac{a}{c^{2}} .
\end{gathered}
$$

Proof. Theorem 15 follows from Theorems 10, 12, and 14.

Remark 6. In the paper of Gao and Lee [14], an asymptotic formula with four terms and an error estimate of order

$$
O\left((\log K)^{-\frac{3}{4}}\right), \quad K \rightarrow \infty
$$

was found for the implied volatility in the negatively correlated Heston model (see [14, Cor. 8.1]). Using (30) and Theorem 14, we can obtain a sharper asymptotic formula with five terms and an error estimate of order $O\left((\log K)^{-1}\right)$ as $K \rightarrow \infty$. The fifth term in this formula is of the form $c \frac{\log \log K}{\log K}$. The previous expression tends to zero faster than the expression in (49). The reason why the formula obtained in [14] contains a weaker error estimate is the following. In the proof of their result, Gao and Lee used formula (4.2) from [13], which is an asymptotic formula for the call pricing function in the Heston model with a relative error estimate $O\left((\log K)^{-\frac{1}{4}}\right)$. However, formula (4.2) in [13] in our opinion contains a typo. More precisely, the power $-\frac{1}{4}$ in the error estimate in that formula can be replaced by the power $-\frac{1}{2}$. Indeed, it suffices to integrate the expressions in formula (4.1) in [13] twice. Formula (4.1) is an asymptotic formula for the stock price density in the negatively correlated Heston model, containing a correct relative error estimate $O\left((\log x)^{-\frac{1}{2}}\right)$. In the presentation of the results from [13] in the book [17], the asymptotic formula for the call pricing function in the negatively correlated Heston model contains a correct error term (see formula (8.28) in [17]). 
Remark 7. The asymptotic behavior of the implied volatility at large strikes in Kou's model was studied in [29] and [15]. Since Kou's model is the Black-Scholes model with double exponential jumps, the jump part always dominates. Indeed, the decay of the stock price density in the Black-Scholes model is log-normal, while the density of the exponential Lévy part of Kou's model decays as a regularly varying function. The authors of [15] obtain an asymptotic formula for the implied volatility with four terms and an error estimate of order $O\left((\log K)^{-\frac{3}{4}}\right)$ as in [14]. It is not hard to obtain a similar expansion with five terms and an error estimate of order $O\left((\log K)^{-1}\right)$ as $K \rightarrow \infty$, using Theorems 8, 19, and 14 established in the present paper.

Our next goal is to characterize the asymptotic behavior of the implied volatility at small strikes. Here we borrow various ideas used in section 9.7 of [17]. For the sake of brevity, we again assume that $x_{0}=1$. Set $G(K)=K P\left(K^{-1}\right)$. Then $G$ is a call pricing function. The corresponding marginal densities are as follows:

$$
\widetilde{D}_{T}(x)=x^{-3} D_{T}\left(x^{-1}\right)
$$

(see Remark 9.20 in [17]). Now, using Theorems 11 and 13, formula (50), and the fact that $I(K)=I_{G}\left(K^{-1}\right)$ (see Lemma 9.23 in [17]), we obtain the following assertion.

Theorem 16. Let $T>0$, and suppose $\widetilde{A}_{3}>\eta_{2}-1$. Then the following formula holds for the implied volatility in the Heston model with double exponential jumps:

$$
\begin{aligned}
& I(K)=\frac{\sqrt{2}}{\sqrt{T}}\left(\sqrt{s_{3}+1}-\sqrt{s_{3}}\right) \sqrt{\log \frac{x_{0}}{K}}+\frac{s_{2}}{\sqrt{2 T}}\left(\frac{1}{\sqrt{s_{3}}}-\frac{1}{\sqrt{s_{3}+1}}\right) \\
& +\frac{2 s_{4}+1}{2 \sqrt{2 T}}\left(\frac{1}{\sqrt{s_{3}}}-\frac{1}{\sqrt{s_{3}+1}}\right) \frac{\log \log \frac{x_{0}}{K}}{\sqrt{\log \frac{x_{0}}{K}}} \\
& +\left[\frac{1}{\sqrt{2 T}} \log \frac{\sqrt{s_{3}+1} \sqrt{s_{3}}\left(\sqrt{s_{3}+1}-\sqrt{s_{3}}\right)}{2 \sqrt{\pi} s_{1}}+\frac{s_{2}^{2}}{4 \sqrt{2 T}}\left(\frac{1}{s_{3}^{\frac{3}{2}}}-\frac{1}{\left(s_{3}+1\right)^{\frac{3}{2}}}\right)\right] \frac{1}{\sqrt{\log \frac{x_{0}}{K}}} \\
& +\frac{s_{2}\left(2 s_{4}+1\right)}{4 \sqrt{2 T}}\left(\frac{1}{s_{3}^{\frac{3}{2}}}-\frac{1}{\left(s_{3}+1\right)^{\frac{3}{2}}}\right) \frac{\log \log \frac{x_{0}}{K}}{\log \frac{x_{0}}{K}}+O\left(\frac{1}{\log \frac{x_{0}}{K}}\right)
\end{aligned}
$$

as $K \rightarrow 0$. In (51),

$$
\begin{gathered}
s_{1}=\frac{1}{2 \sqrt{\pi}} m_{-\eta_{2}}\left(D_{T}^{(1)}\right)\left(\eta_{2} \lambda T q\right)^{\frac{1}{4}} \exp \left\{\frac{\eta_{1} \lambda T p}{\eta_{1}+\eta_{2}}-\lambda T\right\}, \\
s_{2}=2 \sqrt{\eta_{2} \lambda T q}, \quad s_{3}=\eta_{2}, \quad \text { and } \quad s_{4}=-\frac{3}{4} .
\end{gathered}
$$

On the other hand, if $\widetilde{A}_{3}<\eta_{2}-1$, then (51) holds with

$$
\begin{gathered}
s_{1}=\left[e^{-\lambda T}+m_{A_{3}-1}(H(T, \cdot))\right] \widetilde{B}_{1}, \\
s_{2}=\widetilde{A}_{2}, \quad s_{3}=\widetilde{A}_{3}+1, \quad \text { and } \quad s_{4}=-\frac{3}{4}+\frac{a}{c^{2}} .
\end{gathered}
$$


5. Proofs of the main results. Our main objective in the present section is to prove Theorems 10-13. We first establish several preliminary results, which are of independent interest. The proofs of Theorems 10-13 will be completed at the end of subsection 5.3.

Set $T_{t}=\sum_{i=1}^{N_{t}} U_{i}$ (see section 3 for the definition of $N_{t}$ and $U_{i}$ ). The distribution $\mu_{t}$ of the random variable $T_{t}$ is given by

$$
d \mu_{t}(y)=e^{-\lambda t} d \delta_{0}(y)+\left[G_{1}(t, y) e^{-\eta_{1} y} \chi_{\{y>0\}}+G_{2}(t, y) e^{\eta_{2} y} \chi_{\{y<0\}}\right] d y,
$$

where $y \in(-\infty, \infty)$ and $\delta_{0}$ is the delta-measure at $y=0$. The functions $G_{1}$ and $G_{2}$ in the previous formula are defined by

$$
G_{1}(t, u)=\sum_{k=0}^{\infty} a_{k} u^{k}, \quad u>0
$$

with

$$
a_{k}=\frac{\eta_{1}^{k+1}}{k !} \sum_{n=k+1}^{\infty} \pi_{n} P_{n, k+1}
$$

and

$$
G_{2}(t,-u)=\sum_{k=0}^{\infty} b_{k} u^{k}, \quad u>0
$$

with

$$
b_{k}=\frac{\eta_{2}^{k+1}}{k !} \sum_{n=k+1}^{\infty} \pi_{n} Q_{n, k+1}
$$

The numbers $\pi_{n}$ in the previous formulas depend on $t$. They are defined by $\pi_{0}=e^{-\lambda t}$ and $\pi_{n}=e^{-\lambda t}(\lambda t)^{n}(n !)^{-1}$ for all $n \geq 1$. In addition, the numbers $P_{n, k}$ and $Q_{n, k}$ are given by

$$
P_{n, k}=\sum_{i=k}^{n-1}\left(\begin{array}{c}
n-k-1 \\
i-k
\end{array}\right)\left(\begin{array}{c}
n \\
i
\end{array}\right)\left(\frac{\eta_{1}}{\eta_{1}+\eta_{2}}\right)^{i-k}\left(\frac{\eta_{2}}{\eta_{1}+\eta_{2}}\right)^{n-i} p^{i} q^{n-i}
$$

for all $1 \leq k \leq n-1$, and

$$
Q_{n, k}=\sum_{i=k}^{n-1}\left(\begin{array}{c}
n-k-1 \\
i-k
\end{array}\right)\left(\begin{array}{c}
n \\
i
\end{array}\right)\left(\frac{\eta_{1}}{\eta_{1}+\eta_{2}}\right)^{n-i}\left(\frac{\eta_{2}}{\eta_{1}+\eta_{2}}\right)^{i-k} p^{n-i} q^{i}
$$

for all $1 \leq k \leq n-1$. We also have $P_{n, n}=p^{n}$ and $Q_{n, n}=q^{n}$. Formula (52) can be derived using Proposition B.1 in [22] (see the derivation in [19] or in section 10.8 of [17]).

It follows from (52) that the distribution $\mu_{t}^{(2)}$ of the random variable $X_{t}^{(2)}$ satisfies

$$
d \mu_{t}^{(2)}(x)=e^{-\lambda t} d \delta_{1}(x)+H(t, x) d x, \quad x>0 .
$$


In (57), $\delta_{1}$ is the delta-measure at $x=1$, the function $H$ is defined by

$$
H(t, x)=H_{1}(t, x) x^{-\eta_{1}-1} \chi_{\{x>1\}}+H_{2}(t, x) x^{\eta_{2}-1} \chi_{\{0<x<1\}},
$$

where

$$
H_{1}(t, x)=G_{1}(t, \log x), \quad x>1,
$$

and

$$
H_{2}(t, x)=G_{2}(t, \log x), \quad 0<x<1 .
$$

The next assertion provides useful approximations to the coefficients $a_{k}$ and $b_{k}$ appearing in (54) and (55).

Theorem 17. There exist positive constants $c_{1}$ and $c_{2}$, independent of $k$, and such that

$$
0<a_{k}-\widehat{a}_{k} \leq c_{1} \frac{\widehat{a}_{k}}{k+1}, \quad k \geq 0,
$$

and

$$
0<b_{k}-\widehat{b}_{k} \leq c_{2} \frac{\widehat{b}_{k}}{k+1}, \quad k \geq 0
$$

where

$$
\widehat{a}_{k}=\exp \left\{\frac{\eta_{2} \lambda t q}{\eta_{1}+\eta_{2}}-\lambda t\right\} \frac{\left(\eta_{1} \lambda t p\right)^{k+1}}{k !(k+1) !}, \quad k \geq 0,
$$

and

$$
\widehat{b}_{k}=\exp \left\{\frac{\eta_{1} \lambda t p}{\eta_{1}+\eta_{2}}-\lambda t\right\} \frac{\left(\eta_{2} \lambda t q\right)^{k+1}}{k !(k+1) !}, \quad k \geq 0 .
$$

Proof. For $i \geq 1$ and $m \geq i+1$, put

$$
\gamma_{k+m, k+i}=\left(\begin{array}{c}
m-2 \\
i-1
\end{array}\right)\left(\begin{array}{c}
k+m \\
k+i
\end{array}\right)\left(\frac{\eta_{1}}{\eta_{1}+\eta_{2}}\right)^{i-1}\left(\frac{\eta_{2}}{\eta_{1}+\eta_{2}}\right)^{m-i} p^{k+i} q^{m-i}
$$

It follows from (54) and (56) that for all $k \geq 1$,

$$
a_{k}=\sum_{i=0}^{\infty} a_{k, i}
$$

where

$$
a_{k, 0}=\frac{\eta_{1}^{k+1}}{k !} \pi_{k+1} p^{k+1}
$$

and

$$
a_{k, i}=\frac{\eta_{1}^{k+1}}{k !} \sum_{m=i+1}^{\infty} \pi_{k+m} \gamma_{k+m, k+i}
$$


for all $i \geq 1$. We have

$$
\begin{aligned}
a_{k, 0}+a_{k, 1} & =e^{-\lambda t} \frac{\left(\eta_{1} \lambda t p\right)^{k+1}}{k !(k+1) !}\left[1+\sum_{m=2}^{\infty} \frac{1}{(m-1) !}\left(\frac{\eta_{2} \lambda t q}{\eta_{1}+\eta_{2}}\right)^{m-1}\right] \\
& =\exp \left\{\frac{\eta_{2} \lambda t q}{\eta_{1}+\eta_{2}}-\lambda t\right\} \frac{\left(\eta_{1} \lambda t p\right)^{k+1}}{k !(k+1) !}=\widehat{a}_{k}
\end{aligned}
$$

Therefore, (63), (64), and (65) imply the following:

$$
\begin{aligned}
0 \leq & a_{k}-\widehat{a}_{k}=\sum_{i=2}^{\infty} a_{k, i} \\
= & e^{-\lambda t} \frac{\left(\eta_{1} \lambda t p\right)^{k+1}}{k !(k+1) !} \sum_{i=2}^{\infty} \frac{1}{(k+i) \cdots(k+2)(i-1) !}\left(\frac{\eta_{1}}{\eta_{1}+\eta_{2}}\right)^{i-1} p^{i-1} \\
& \sum_{m=i+1}^{\infty} \frac{(\lambda t)^{m-1}(m-2) !}{(m-i-1) !(m-i) !}\left(\frac{\eta_{2}}{\eta_{1}+\eta_{2}}\right)^{m-i} q^{m-i} \\
\leq & \beta_{1} \frac{\widehat{a}_{k}}{k+1} \sum_{i=2}^{\infty} \frac{1}{i !(i-1) !}\left(\frac{\eta_{1}}{\eta_{1}+\eta_{2}}\right)^{i-1}(\lambda t p)^{i-1} \sum_{j=0}^{\infty} \frac{(\lambda t)^{j+1}(j+i-1) !}{j !(j+1) !}\left(\frac{\eta_{2}}{\eta_{1}+\eta_{2}}\right)^{j+1} q^{j+1},
\end{aligned}
$$

where $\beta_{1}$ is a positive constant. For $i \geq 2$ and $j \geq 1$, we have

$$
\frac{(j+i-1) !}{i ! j !} \leq \frac{(j+i)^{i}}{i !} \leq \beta_{2} e^{i}\left(1+\frac{j}{i}\right)^{i} \leq \beta_{2} e^{i+j}
$$

where $\beta_{2}$ is a positive constant. In the proof of the previous estimates, we used Stirling's formula. Now, it is not hard to see that the last double series in (66) converges, and it follows from (66) that the estimate in (61) is valid. The proof of the estimate in (62) is similar.

This completes the proof of Theorem 17.

We will next further simplify formulas (61) and (62). Set

$$
\begin{array}{ll}
C_{1}=\frac{\eta_{1} \lambda t p}{2 \pi} \exp \left\{\frac{\eta_{2} \lambda t q}{\eta_{1}+\eta_{2}}-\lambda t\right\}, & B_{1}=\eta_{1} \lambda t p, \\
C_{2}=\frac{\eta_{2} \lambda t q}{2 \pi} \exp \left\{\frac{\eta_{1} \lambda t p}{\eta_{1}+\eta_{2}}-\lambda t\right\}, & B_{2}=\eta_{2} \lambda t q,
\end{array}
$$

and consider the following sequences:

$$
d_{0}=C_{1}, \quad d_{k}=C_{1} \frac{B_{1}^{k} e^{2 k}}{k^{2 k+2}}, \quad k \geq 1,
$$

and

$$
l_{0}=C_{2}, \quad l_{k}=C_{2} \frac{B_{2}^{k} e^{2 k}}{k^{2 k+2}}, \quad k \geq 1 .
$$


Corollary 2. The following formulas hold:

$$
\left|a_{k}-d_{k}\right| \leq \frac{\alpha_{1}}{k+1} d_{k}, \quad k \geq 0,
$$

and

$$
\left|b_{k}-l_{k}\right| \leq \frac{\alpha_{2}}{k+1} l_{k}, \quad k \geq 0,
$$

where $\alpha_{1}$ and $\alpha_{2}$ are some positive constants.

Proof. We will need Stirling's formula in the asymptotic form as follows:

$$
n !=\sqrt{2 \pi} n^{n+\frac{1}{2}} e^{-n}\left(1+O\left(\frac{1}{n}\right)\right)
$$

as $n \rightarrow \infty$. It is not hard to see, using (61) and (70), that

$$
\begin{aligned}
a_{k} & =\exp \left\{\frac{\eta_{2} \lambda t q}{\eta_{1}+\eta_{2}}-\lambda t\right\} \frac{\left(\eta_{1} \lambda t p\right)^{k+1}}{k ! k ! k\left(1+\frac{1}{k+1}\right)}\left(1+O\left(\frac{1}{k}\right)\right) \\
& =\exp \left\{\frac{\eta_{2} \lambda t q}{\eta_{1}+\eta_{2}}-\lambda t\right\} \frac{\left(\eta_{1} \lambda t p\right)^{k+1}}{k ! k ! k}\left(1+O\left(\frac{1}{k+1}\right)\right) \\
& =\frac{1}{2 \pi} \exp \left\{\frac{\eta_{2} \lambda t q}{\eta_{1}+\eta_{2}}-\lambda t\right\} \frac{\left(\eta_{1} \lambda t p\right)^{k+1} e^{2 k}}{k^{2 k+2}}\left(1+O\left(\frac{1}{k+1}\right)\right)
\end{aligned}
$$

as $k \rightarrow \infty$. This establishes (68). The proof of (69) is similar.

5.1. Properties of the functions $H_{1}$ and $H_{2}$. In the present subsection, we study the asymptotic behavior of the functions $H_{1}$ and $H_{2}$ defined in (59) and (60). It will be shown first that these functions are of slow variation with remainder.

Lemma 1. For every $t>0$, the functions $x \mapsto H_{1}(t, x)$ and $x \mapsto H_{2}\left(t, x^{-1}\right)$ belong to the Zygmund class $\mathcal{Z}$.

Proof. Let us fix $t>0$. Since the function $x \mapsto H_{1}(t, x)$ is increasing on $x>1$, the function $\phi_{\alpha}(t, x)=x^{\alpha} H_{1}(t, x)$, where $\alpha>0$, is also increasing. It remains to prove that the function $\psi_{\alpha}(t, x)=x^{-\alpha} H_{1}(t, x)$ is ultimately decreasing. We have

$$
\psi_{\alpha}^{\prime}(t, x)=-\alpha x^{-\alpha-1} G_{1}(t, \log x)+x^{-\alpha-1} G_{1}^{\prime}(t, \log x) .
$$

Therefore, the condition $\psi_{\alpha}^{\prime}(t, x) \leq 0$ is equivalent to the condition

$$
\frac{G_{1}^{\prime}(t, \log x)}{G_{1}(t, \log x)} \leq \alpha
$$

for all $x>x_{\alpha}$, which in turn is equivalent to the condition

$$
\frac{G_{1}^{\prime}(t, \log x)}{G_{1}(t, \log x)} \rightarrow 0
$$

as $x \rightarrow \infty$. Now it is clear that it suffices to prove (71). 
Using the definition of the function $G_{1}$, we obtain

$$
\frac{G_{1}^{\prime}(t, \log x)}{G_{1}(t, \log x)}=\frac{\sum_{k=1}^{\infty} a_{k} k(\log x)^{k-1}}{\sum_{k=0}^{\infty} a_{k}(\log x)^{k}}
$$

where the coefficients $a_{k}$ are defined by (54). It is not hard to see, using (61), that for all $k \geq 2$,

$$
\frac{k a_{k}}{a_{k-1}} \leq \frac{c}{k+1}
$$

with some $c>0$. Hence, for every $\varepsilon>0$ there exists a positive integer $k_{\varepsilon}$ such that $k a_{k} \leq \varepsilon a_{k-1}$ for all $k>k_{\varepsilon}$. It follows from (72) that

$$
\frac{G_{1}^{\prime}(t, \log x)}{G_{1}(t, \log x)} \leq \frac{\sum_{k=1}^{k_{\varepsilon}} a_{k} k(\log x)^{k-1}}{\sum_{k=1}^{\infty} a_{k}(\log x)^{k}}+\varepsilon .
$$

It is clear that for fixed $\varepsilon$ the first term on the right-hand side of (74) tends to 0 as $x \rightarrow \infty$. Now, it is not hard to see that condition (71) holds.

This completes the proof of Lemma 1 for the function $x \mapsto H_{1}(t, x)$. The proof for the function $x \mapsto H_{2}\left(t, \frac{1}{x}\right)$ is similar.

Remark 8. It follows from (57), the fact that $\mathcal{Z} \subset R_{0}$, and Lemma 1 that the process $t \mapsto X_{t}^{(2)}$ is an integrable process.

Lemma 2. For every $t>0$, the functions $x \mapsto H_{1}(t, x)$ and $x \mapsto H_{2}\left(t, x^{-1}\right)$ belong to the class $R_{0}^{g}$, where the function $g$ is given by $g(x)=(\log x)^{-\frac{1}{2}}$.

Proof. The function $x \mapsto H_{1}(t, x)$ is an increasing differentiable function from the Zygmund class. Therefore, it suffices to prove that there exists $\widetilde{c}>0$ such that

$$
\frac{x H_{1}^{\prime}(t, x)}{H_{1}(t, x)} \leq \widetilde{c}(\log x)^{-\frac{1}{2}}
$$

for all $x>x_{0}$ (see Corollary 1 and (10)). It is easy to see that the estimate in (75) is equivalent to

$$
\frac{G_{1}^{\prime}(t, \log x)}{G_{1}(t, \log x)}=O\left((\log x)^{-\frac{1}{2}}\right)
$$

as $x \rightarrow \infty$.

We have

$$
\begin{aligned}
& \frac{G_{1}^{\prime}(t, \log x)}{G_{1}(t, \log x)}=(\log x)^{-1} \frac{\sum_{k=1}^{\infty} a_{k} k(\log x)^{k}}{\sum_{k=0}^{\infty} a_{k}(\log x)^{k}} \\
& =(\log x)^{-1} \frac{\sum_{k=1}^{[\sqrt{\log x}]} a_{k} k(\log x)^{k}+\sum_{k=[\sqrt{\log x}+1}^{\infty} a_{k} k(\log x)^{k}}{\sum_{k=0}^{\infty} a_{k}(\log x)^{k}} \\
& \leq(\log x)^{-\frac{1}{2}}+(\log x)^{-1} \frac{\sum_{k=[\sqrt{\log x}+1}^{\infty} a_{k} k(\log x)^{k}}{\sum_{k=0}^{\infty} a_{k}(\log x)^{k}} .
\end{aligned}
$$


Next, using (73) in (77), we obtain

$$
\begin{aligned}
& \frac{G_{1}^{\prime}(t, \log x)}{G_{1}(t, \log x)} \leq(\log x)^{-\frac{1}{2}}+(\log x)^{-1} \frac{\sum_{k=[\sqrt{\log x}+1}^{\infty} \frac{c a_{k-1}}{k+1}(\log x)^{k}}{\sum_{k=1}^{\infty} a_{k}(\log x)^{k}} \\
& \leq(\log x)^{-\frac{1}{2}}+c(\log x)^{-\frac{3}{2}} \frac{\sum_{k=[\sqrt{\log x}+1}^{\infty} a_{k-1}(\log x)^{k}}{\sum_{k=1}^{\infty} a_{k}(\log x)^{k}} \\
& =O\left((\log x)^{-\frac{1}{2}}\right)
\end{aligned}
$$

as $x \rightarrow \infty$. This establishes estimate (76).

The proof of Lemma 2 in the case of the function $x \mapsto H_{1}(t, x)$ is thus completed. For the function $x \mapsto H_{2}\left(t, \frac{1}{x}\right)$, the proof is similar.

Recall that $G_{1}(t, \cdot)=\sum_{k=0}^{\infty} a_{k} u^{k}$ (see (53)), where the coefficients $a_{k}$ are given by (54). Define two auxiliary functions $\widetilde{G}_{1}(t, \cdot)$ and $\widehat{G}_{1}(t, \cdot)$ as

$$
\widetilde{G}_{1}(t, u)=\sum_{k=0} d_{k} u^{k} \quad \text { and } \quad \widehat{G}_{1}(t, u)=\sum_{k=0}^{\infty} \frac{d_{k}}{k+1} u^{k},
$$

where $u \geq 0$ and the sequence $d$ is given by (67). Then (68) implies that

$$
\left|G_{1}(t, u)-\widetilde{G}_{1}(t, u)\right| \leq \alpha_{1} \widehat{G}_{1}(t, u) .
$$

The functions $\widetilde{G}_{1}$ and $\widehat{G}_{1}$ are defined as sums of certain power series. Our next goal is to compare these functions with some standard functions. Analyzing the structure of the coefficients $d_{k}$, we guess that the following family of functions may be useful:

$$
\lambda_{s, r}(u)=s \cosh (r \sqrt{u})=\sum_{k=0}^{\infty} \widetilde{d}_{k} u^{k}, \quad u \geq 0, \quad r>0, \quad s>0,
$$

where

$$
\widetilde{d}_{k}=s \frac{r^{2 k}}{(2 k) !} .
$$

It is clear that $\widetilde{d}_{0}=s$. Moreover, using Stirling's formula, we see that

$$
\tilde{d}_{k}=s \frac{r^{2 k} e^{2 k}}{2 \sqrt{\pi} 2^{2 k} k^{2 k+\frac{1}{2}}}\left(1+O\left(\frac{1}{k}\right)\right)
$$

as $k \rightarrow \infty$. Next, comparing the coefficients $d_{k}$ and $\widetilde{d}_{k}$, we see that if we set

$$
s=2 \sqrt{\pi} C_{1} \text { and } r=2 \sqrt{B_{1}},
$$

then

$$
\left|d_{k}-(k+1)^{-\frac{3}{2}} \widetilde{d}_{k}\right| \leq \delta_{1}(k+1)^{-\frac{5}{2}} \widetilde{d}_{k}
$$

for some $\delta_{1}>0$ and all $k \geq 0$. Finally, it follows from (68) and (80) that the coefficients $a_{k}$ and $\widetilde{d}_{k}$ satisfy the condition

$$
\left|a_{k}-(k+1)^{-\frac{3}{2}} \widetilde{d}_{k}\right| \leq \delta_{2}(k+1)^{-\frac{5}{2}} \widetilde{d}_{k}
$$

for some $\delta_{2}>0$ and all $k \geq 0$. 
5.2. The Riemann-Liouville integrals. In this subsection, we consider only fractional integrals of functions given by everywhere convergent power series with positive coefficients. Let $f(u)=\sum_{n=0}^{\infty} c_{n} u^{n}$ be a function on $\mathbb{R}$ such that $c_{n}>0$ for all $n \geq 0$ and the function $F(z)=\sum_{n=0}^{\infty} c_{n} z^{n}$ is an entire function on $\mathbb{C}$. For $\alpha<0$, the Riemann-Liouville fractional integral $D^{\alpha} f$ is defined as follows:

$$
D^{\alpha} f(u)=\frac{1}{\Gamma(-\alpha)} \int_{0}^{u} f(y)(u-y)^{-\alpha-1} d y .
$$

Then the following formula is valid:

$$
D^{\alpha} f(u)=u^{-\alpha} \sum_{n=0}^{\infty} c_{n, \alpha} c_{n} u^{n},
$$

where

$$
c_{n, \alpha}=\frac{\Gamma(n+1)}{\Gamma(n-\alpha+1)}
$$

(see the definition of the Riemann-Liouville integral in [24, sect. 5] and [24, sect. 3, formula (3.2)]). We will next characterize the asymptotic behavior of the sequence $c_{n, \alpha}$. Using the asymptotic formula for the Gamma function, that is, the formula

$$
\Gamma(u)=\sqrt{2 \pi} u^{u-\frac{1}{2}} e^{-u}\left(1+O\left(\frac{1}{u}\right)\right)
$$

as $u \rightarrow \infty$, we obtain

$$
\begin{aligned}
c_{n, \alpha} & =\frac{e^{|\alpha|}(n+1)^{n+|\alpha|+\frac{1}{2}}}{(n+|\alpha|+1)^{n+|\alpha|+\frac{1}{2}}(n+1)^{|\alpha|}}\left(1+O\left(\frac{1}{n}\right)\right) \\
& =e^{|\alpha|}\left(\frac{n+1}{n+|\alpha|+1}\right)^{n+|\alpha|+1} \frac{(n+|\alpha|+1)^{\frac{1}{2}}}{(n+1)^{\frac{1}{2}}} \frac{1}{(n+1)^{|\alpha|}}\left(1+O\left(\frac{1}{n}\right)\right) \\
& =e^{|\alpha|}\left(1-\frac{|\alpha|}{n+|\alpha|+1}\right)^{n+|\alpha|+1} \frac{1}{(n+1)^{|\alpha|}}\left(1+O\left(\frac{1}{n}\right)\right)
\end{aligned}
$$

as $n \rightarrow \infty$.

It is not hard to prove that for every $c>0$,

$$
\left(1-\frac{c}{x}\right)^{x}=e^{-c}\left(1+O\left(\frac{1}{x}\right)\right)
$$

as $x \rightarrow \infty$. Therefore,

$$
c_{n, \alpha}=\frac{1}{(n+1)^{|\alpha|}}\left(1+O\left(\frac{1}{n+1}\right)\right)
$$

as $n \rightarrow \infty$, and hence

$$
\frac{1}{(n+1)^{|\alpha|}}=c_{n, \alpha}\left(1+O\left(\frac{1}{n+1}\right)\right)
$$


as $n \rightarrow \infty$. Since

$$
\frac{c_{n, \alpha}}{n+1}=\frac{\Gamma(n+1)}{(n+1) \Gamma(n+|\alpha|+1)} \leq c \frac{\Gamma(n+1)}{(n+|\alpha|+1) \Gamma(n+|\alpha|+1)}=c \frac{\Gamma(n+1)}{\Gamma(n+|\alpha|+2)}=c_{n, \alpha-1},
$$

formula (84) implies that there exists a constant $\delta_{3}>0$ for which

$$
\left|\frac{1}{(n+1)^{|\alpha|}}-c_{n, \alpha}\right| \leq \delta_{3} c_{n, \alpha-1}
$$

for all $n \geq 0$.

Our next goal is to combine various estimates for the coefficients obtained above.

Lemma 3. There exists a constant $\delta_{4}>0$ such that

$$
\left|a_{k}-c_{k,-\frac{3}{2}} \widetilde{d}_{k}\right| \leq \delta_{4} c_{k,-\frac{5}{2}} \widetilde{d}_{k}
$$

for all $k \geq 0$.

Proof. It follows from (81), (84), and (85) that

$$
\begin{aligned}
& \left|a_{k}-c_{k,-\frac{3}{2}} \widetilde{d}_{k}\right| \leq\left|a_{k}-(k+1)^{-\frac{3}{2}} \widetilde{d}_{k}\right|+\left|c_{k,-\frac{3}{2}}-(k+1)^{-\frac{3}{2}}\right| \widetilde{d}_{k} \\
& \leq \delta_{2}(k+1)^{-\frac{5}{2}} \widetilde{d}_{k}+\delta_{3} c_{k,-\frac{5}{2}} \widetilde{d}_{k} \leq \delta_{4} c_{k,-\frac{5}{2}} \widetilde{d}_{k} .
\end{aligned}
$$

This completes the proof of Lemma 3.

The following assertion can be easily derived from (53), (78), and (83).

Theorem 18. There exists a positive constant $c$ such that

$$
\left|G_{1}(t, u)-u^{-\frac{3}{2}} D^{-\frac{3}{2}} \lambda_{s, r}(u)\right| \leq c u^{-\frac{5}{2}} D^{-\frac{5}{2}} \lambda_{s, r}(u)
$$

for all $u>0$. In (86), the values of the parameters $r$ and $s$ are chosen according to (79).

It follows from Theorem 18 that in order to understand the asymptotic behavior of the function $G_{1}(t, u)$ as $u \rightarrow \infty$, we have to study how the fractional integrals appearing in (86) behave for large values of $u$. Using (78) and (82), we obtain

$$
\begin{aligned}
& u^{-\frac{3}{2}} D^{-\frac{3}{2}} \lambda_{s, r}(u)=\frac{s}{\Gamma\left(\frac{3}{2}\right)} \int_{0}^{1} \cosh (r \sqrt{y u})(1-y)^{\frac{1}{2}} d y \\
& =\frac{s}{\Gamma\left(\frac{3}{2}\right)} \int_{0}^{1} e^{r \sqrt{u} z} z\left(1-z^{2}\right)^{\frac{1}{2}} d z+\frac{s}{\Gamma\left(\frac{3}{2}\right)} \int_{0}^{1} e^{-r \sqrt{u} z} z\left(1-z^{2}\right)^{\frac{1}{2}} d z
\end{aligned}
$$

and

$$
\begin{aligned}
& u^{-\frac{5}{2}} D^{-\frac{5}{2}} \lambda_{s, r}(u)=\frac{s}{\Gamma\left(\frac{5}{2}\right)} \int_{0}^{1} \cosh (r \sqrt{y u})(1-y)^{\frac{3}{2}} d y \\
& =\frac{s}{\Gamma\left(\frac{5}{2}\right)} \int_{0}^{1} e^{r \sqrt{u} z} z\left(1-z^{2}\right)^{\frac{3}{2}} d z+\frac{s}{\Gamma\left(\frac{5}{2}\right)} \int_{0}^{1} e^{-r \sqrt{u} z} z\left(1-z^{2}\right)^{\frac{3}{2}} d z .
\end{aligned}
$$


We have

$$
\int_{0}^{1} e^{-r \sqrt{u} z} z\left(1-z^{2}\right)^{\frac{1}{2}} d z=\int_{0}^{\frac{1}{2}}+\int_{\frac{1}{2}}^{1}=O\left(u^{-1}\right) .
$$

Indeed, the third integral in (89) is $O\left(e^{-\frac{1}{2} r \sqrt{u}}\right)$, while the second integral can be estimated, using the integration by parts twice. Similarly,

$$
\int_{0}^{1} e^{-r \sqrt{u} z} z\left(1-z^{2}\right)^{\frac{3}{2}} d z=O\left(u^{-1}\right)
$$

as $u \rightarrow \infty$. In addition,

$\int_{0}^{1} e^{r \sqrt{u} z} z\left(1-z^{2}\right)^{\frac{1}{2}} d z=e^{r \sqrt{u}} \int_{0}^{1} e^{r \sqrt{u}(z-1)} z\left(1-z^{2}\right)^{\frac{1}{2}} d z=e^{r \sqrt{u}} \int_{0}^{1} e^{-r \sqrt{u} y}(1-y)(2-y)^{\frac{1}{2}} y^{\frac{1}{2}} d y$.

For small values of $y$, we have $(1-y)(2-y)^{\frac{1}{2}} y^{\frac{1}{2}}=\sqrt{2} y^{\frac{1}{2}}+c_{1} y^{\frac{3}{2}}+\cdots$. Using Watson's lemma (see $[10$, p. 103]), we obtain

$$
\int_{0}^{1} e^{r \sqrt{u} z} z\left(1-z^{2}\right)^{\frac{1}{2}} d z=e^{r \sqrt{u}}\left[\sqrt{2} \Gamma\left(\frac{3}{2}\right) r^{-\frac{3}{2}} u^{-\frac{3}{4}}+O\left(u^{-\frac{5}{4}}\right)\right]
$$

as $y \rightarrow \infty$. Similarly,

$$
\int_{0}^{1} e^{r \sqrt{u} z} z\left(1-z^{2}\right)^{\frac{3}{2}} d z=e^{r \sqrt{u}}\left[2^{\frac{3}{2}} \Gamma\left(\frac{5}{2}\right) r^{-\frac{5}{2}} u^{-\frac{5}{4}}+O\left(u^{-\frac{7}{4}}\right)\right]
$$

as $y \rightarrow \infty$.

5.3. Completion of the proofs of the main results. We will first formulate and prove one of the main technical results of the present paper.

Theorem 19. Let $H_{1}(t, \cdot)$ and $H_{2}(t, \cdot)$ be the functions defined by (59) and (60), respectively. Then the following asymptotic formulas hold:

$$
\begin{aligned}
H_{1}(t, x)= & \frac{1}{2 \sqrt{\pi}}\left(\eta_{1} \lambda t p\right)^{\frac{1}{4}} \exp \left\{\frac{\eta_{2} \lambda t q}{\eta_{1}+\eta_{2}}-\lambda t\right\}(\log x)^{-\frac{3}{4}} \\
& \times \exp \left\{2 \sqrt{\eta_{1} \lambda t p} \sqrt{\log x}\right\}\left(1+O\left((\log x)^{-\frac{1}{2}}\right)\right)
\end{aligned}
$$

as $x \rightarrow \infty$, and

$$
\begin{aligned}
H_{2}(t, x)= & \frac{1}{2 \sqrt{\pi}}\left(\eta_{2} \lambda t q\right)^{\frac{1}{4}} \exp \left\{\frac{\eta_{1} \lambda t p}{\eta_{1}+\eta_{2}}-\lambda t\right\}\left(\log \frac{1}{x}\right)^{-\frac{3}{4}} \\
& \times \exp \left\{2 \sqrt{\eta_{2} \lambda t q} \sqrt{\log \frac{1}{x}}\right\}\left(1+O\left(\left(\log \frac{1}{x}\right)^{-\frac{1}{2}}\right)\right)
\end{aligned}
$$

as $x \rightarrow 0$. 
Proof. Formula (93) follows from formulas (86)-(92). Here we take into account that the values of the parameters $r$ and $s$ are given by (79). The proof of formula (94) uses the same ideas, and we leave it as an exercise for the interested reader.

Theorem 19 allows us to characterize the asymptotic behavior of the absolutely continuous part $H$ of the distribution $\mu_{t}^{(2)}$ of the random variable $X_{t}^{(2)}$. Recall that

$$
d \mu_{t}^{(2)}(x)=e^{-\lambda t} d \delta_{1}(x)+H(t, x) d x, \quad x>0,
$$

where

$$
H(t, x)=H_{1}(t, x) x^{-\eta_{1}-1} \chi_{\{x>1\}}+H_{2}(t, x) x^{\eta_{2}-1} \chi_{\{0<x<1\}}
$$

(see (57)). It is clear from Theorem 19 that the following assertion holds.

Corollary 3. For every $t>0$,

$$
\begin{aligned}
H(t, x)= & \frac{1}{2 \sqrt{\pi}}\left(\eta_{1} \lambda t p\right)^{\frac{1}{4}} \exp \left\{\frac{\eta_{2} \lambda t q}{\eta_{1}+\eta_{2}}-\lambda t\right\}(\log x)^{-\frac{3}{4}} \\
& \times \exp \left\{2 \sqrt{\eta_{1} \lambda t p} \sqrt{\log x}\right\} x^{-\eta_{1}-1}\left(1+O\left((\log x)^{-\frac{1}{2}}\right)\right)
\end{aligned}
$$

as $x \rightarrow \infty$, and

$$
\begin{aligned}
H(t, x)= & \frac{1}{2 \sqrt{\pi}}\left(\eta_{2} \lambda t q\right)^{\frac{1}{4}} \exp \left\{\frac{\eta_{1} \lambda t p}{\eta_{1}+\eta_{2}}-\lambda t\right\}\left(\log \frac{1}{x}\right)^{-\frac{3}{4}} \\
& \times \exp \left\{2 \sqrt{\eta_{2} \lambda t q} \sqrt{\log \frac{1}{x}}\right\} x^{\eta_{2}-1}\left(1+O\left(\left(\log \frac{1}{x}\right)^{-\frac{1}{2}}\right)\right)
\end{aligned}
$$

as $x \rightarrow 0$.

We are finally ready to complete the proof of Theorems 10-13. Recall that we denoted by $D_{t}^{(1)}$ the stock price density in the Heston model. The distribution $\mu_{t}^{(2)}$ of the random variable $\exp \left\{T_{t}\right\}$ has a singular component at $x=1$ (see (57)). However, we can still use a formula similar to formula (1) to estimate $D_{t}$. We have

$$
D_{t}(x)=e^{-\lambda t} D_{t}^{(1)}(x)+D_{t}^{(1)} \stackrel{M}{\star} H(t, \cdot)(x) .
$$

Our next goal is to apply Theorem 8 to characterize the asymptotic behavior of the last term in (97). We put $U(x)=D_{t}^{(1)}(x), \rho=-\eta_{1}-1, l(x)=H_{1}(t, x), f(x)=H(t, x), \sigma=-A_{3}$, $\tau=\widetilde{A}_{3}$, and $h(x)=0$. Then, $\sigma<\rho<\tau$. Indeed, the condition $1+\eta_{1}<A_{3}$ is equivalent to $\sigma<\rho$. In addition, since $\widetilde{A}_{3}>-1$ (use the integrability of the function $D_{t}^{(1)}$ and (31)), we have $\rho<\tau$. Now, taking into account Remark 5 and Lemma 2, we see that the conditions in the formulation of Theorem 8 hold. It follows that

$$
D_{t}^{(1)} \stackrel{M}{\star} H(t, \cdot)(x)=M D_{t}^{(1)}\left(-\eta_{1}-1\right) x^{-\eta_{1}-1} H_{1}(t, x)\left(1+O\left((\log x)^{-\frac{1}{2}}\right)\right)
$$

as $x \rightarrow \infty$. Finally, it is not hard to see that (30), (95), (97), (98), and the condition $1+\eta_{1}<A_{3}$ imply formula (38). 
This completes the proof of Theorem 10 .

The proof of Theorem 11 is similar to that of Theorem 10. It is based on Theorem 9, (31), (58), Lemma 2, (96), and (97). We leave filling in the details to the interested reader.

Theorems 12 and 13 can be derived from formulas (32), (33), and (97), using Theorems 8 and 9.

6. More applications. The example discussed in this section is a stochastic stock price model that is a mixture of the Heston model with a special exponential Lévy model. The log-price process in the perturbing model is the Lévy process with normal inverse Gaussian marginals (the NIG process). We will characterize the asymptotic behavior of the implied volatility at large strikes in the perturbed Heston model mentioned above. The behavior at the small strikes can be characterized similarly.

The normal inverse Gaussian distribution and the NIG process were introduced by Barndorff-Nielsen in [6] and [7], respectively (see also [8]). For the sake of simplicity, we will consider only symmetric centered NIG processes. The general case can be dealt with similarly. Let $\alpha>0$ and $\delta>0$; let $W_{t}^{(\alpha)}=W_{t}+\alpha t, t \geq 0$, be a Brownian motion with drift; and let $A$ be the inverse Gaussian process given by $A_{t}=\inf \left\{s>0: W_{s}^{(\alpha)}=\delta t\right\}$. Consider also an independent standard Brownian motion $\widetilde{W}_{t}, t \geq 0$. Then the NIG process is defined by $Y_{t}=\widetilde{W}_{A_{t}}, t \geq 0$. The parameter $\alpha$ controls the tail heaviness of marginal distributions, while $\delta$ is the scale parameter.

Let us consider a mixed model $X_{t}=X_{t}^{(1)} X_{t}^{(2)}, t \geq 0$, where $X^{(1)}$ is the price process in the Heston model defined in (29), while $X^{(2)}=\exp \left\{Y_{t}\right\}$. As before, we denote by $D_{t}^{(k)}$ the distribution density of the random variable $X_{t}^{(k)}, k=1,2$, and by $\widetilde{D}_{t}^{(2)}$ the density of $Y_{t}$.

There exists a closed-form expression for the density $D_{t}^{(2)}$. The modified Bessel function $K_{1}$ of the third kind will be needed in what follows. This function is defined by

$$
K_{1}(z)=\frac{1}{2} \int_{0}^{\infty} \exp \left\{-\frac{z}{2}\left(u+\frac{1}{u}\right)\right\} d u .
$$

Denote by $(\gamma, 0, \nu)$ the Lévy triplet associated with the process $Y$. It is known that

$$
\gamma=\frac{2 \alpha \delta}{\pi} \int_{0}^{1} K_{1}(\alpha x) d x
$$

and

$$
\nu(d y)=\frac{\alpha \delta}{\pi} \frac{K_{1}(\alpha|y|)}{|y|} d y .
$$

The following formula holds for the density $\widetilde{D}_{t}^{(2)}$ :

$$
\widetilde{D}_{t}^{(2)}(y)=k(t) \frac{K_{1}\left(\alpha \sqrt{y^{2}+\delta^{2} t^{2}}\right)}{\sqrt{y^{2}+\delta^{2} t^{2}}},
$$

where

$$
k(t)=\frac{\alpha \delta t e^{\alpha \delta t}}{\pi} .
$$


Therefore,

$$
D_{t}^{(2)}(x)=\frac{k(t)}{x} \frac{K_{1}\left(\alpha \sqrt{(\log x)^{2}+\delta^{2} t^{2}}\right)}{\sqrt{(\log x)^{2}+\delta^{2} t^{2}}}
$$

for all $x>0$. The previous formulas can be found in [26]. It is known that

$$
K_{1}(z)=\sqrt{\frac{\pi}{2 z}} e^{-z}\left(1+O\left(\frac{1}{z}\right)\right)
$$

as $z \rightarrow \infty$ (see formula 9.7.2 in [1]). It follows that

$$
D_{t}^{(2)}(x)=\frac{k(t)}{x} \sqrt{\frac{\pi}{2 \alpha}} \frac{\exp \left\{-\alpha \sqrt{(\log x)^{2}+\delta^{2} t^{2}}\right\}}{\left((\log x)^{2}+\delta^{2} t^{2}\right)^{\frac{3}{4}}}\left(1+O\left(\frac{1}{\log x}\right)\right)
$$

as $x \rightarrow \infty$.

Since for every $A>0$ and $\alpha>0$,

$$
\frac{1}{\left((\log x)^{2}+A\right)^{\frac{3}{4}}}=\frac{1}{\left((\log x)^{2}\right)^{\frac{3}{4}}}\left(1+O\left(\frac{1}{\log x}\right)\right)
$$

and

$$
\exp \left\{-\alpha \sqrt{(\log x)^{2}+A}\right\}=\exp \left\{-\alpha \sqrt{(\log x)^{2}}\right\}\left(1+O\left(\frac{1}{\log x}\right)\right)
$$

as $x \rightarrow \infty$, formula (99) implies that

$$
D_{t}^{(2)}(x)=k(t) \sqrt{\frac{\pi}{2 \alpha}} x^{-\alpha-1}(\log x)^{-\frac{3}{2}}\left(1+O\left(\frac{1}{\log x}\right)\right)
$$

as $x \rightarrow \infty$.

It is not hard to see, using formulas (100) and (32), that for $A_{3}<\alpha+1$, the Heston part of the mixed model dominates, while for $\alpha+1<A_{3}$, the NIG part dominates. Our next goal is to apply Theorem 14 to the Heston+NIG model. The no-arbitrage condition for this model is

$$
\alpha \geq 1 \quad \text { and } \quad \mu=\delta\left(\sqrt{\alpha^{2}-1}-\alpha\right),
$$

where $\mu$ is the drift parameter in the Heston model. Recall that we assume that $r=0$. Condition (101) can be obtained, using the mean-correcting argument (see the references in the beginning of section 4) and an explicit formula for the characteristic function of the NIG distribution (see [7]; see also [26, sect. 5.39)]).

Theorem 20. Suppose the no-arbitrage condition in (101) holds, and let $A_{3}<\alpha+1$. Then formula (44) is valid for the implied volatility in the Heston + NIG model with

$$
r_{1}=m_{A_{3}-1}\left(D_{T}^{(2)}\right) B_{1},
$$

$r_{2}=A_{2}, r_{3}=A_{3}$, and $r_{4}=-\frac{3}{4}+\frac{a}{c^{2}}$. In addition, if $\alpha+1<A_{3}$, then formula (44) is valid with

$$
r_{1}=k(t) \sqrt{\frac{\pi}{2 \alpha}} m_{\alpha}\left(D_{T}^{(1)}\right),
$$


$r_{2}=0, r_{3}=\alpha+1$, and $r_{4}=-\frac{3}{2}$.

Theorem 20 follows from (32), (100), Theorem 8, and Theorem 14. A similar theorem can be obtained in the case where $K \rightarrow 0$. We leave the formulation and the proof of such a theorem as an exercise for the interested reader.

Remark 9. The methods developed in the present paper are rather universal. They can be used to approximate the stock price density and the implied volatility in many mixed stochastic models. For instance, we can replace the Heston model with jumps by the Stein-Stein model with jumps (see [17] for a discussion of the asymptotic behavior of the stock price density in the Stein-Stein model) and also use jump processes different from the double exponential process or the NIG process. We need only to know appropriate asymptotic formulas with error estimates for the marginal distributions of the jump process, and such formulas are readily available.

Acknowledgments. The authors thank Roger Lee for valuable comments and Stefan Gerhold for providing references [12] and [27].

\section{REFERENCES}

[1] M. Abramovitz And I. A. Stegun, Eds., Handbook of Mathematical Functions, Appl. Math. Ser. 55, National Bureau of Standards, Washington, DC, 1972.

[2] J. M. P. Albin And M. Sunden, On the asymptotic behavior of Lévy processes, Part I, Subexponential and exponential processes, Stochastic Process. Appl., 119 (2009), pp. 281-304.

[3] L. Andersen and A. Lipton, Asymptotics for exponential Lévy processes and their volatility smile: Survey and new results, Int. J. Theor. Appl. Finance, 16 (2013), 1350001.

[4] L. B. G. Andersen and V. V. Piterbarg, Moment explosions in stochastic volatility models, Finance Stoch., 11 (2007), pp. 29-50.

55] D. ARANDElović, Sur un théorème mercerien asymptotique générale pour des fonctions à comportement régulier, Publ. Inst. Math., 20 (1976), pp. 29-36.

[6] O. E. BARndorfF-Nielsen, Exponentially decreasing distributions for the logarithm of particle size, Proc. Roy. Soc. London Ser. A, 353 (1977), pp. 401-419.

[7] O. E. Barndorff-Nielsen, Normal Inverse Gaussian Processes and the Modelling of Stock Returns, Research Report 300, Department of Theoretical Statistics, Aarhus University, Aarhus, Denmark, 1995.

[8] O. E. BARndorff-Nielsen, Normal inverse Gaussian distributions and stochastic volatility modelling, Scand. J. Statist., 24 (1997), pp. 1-13.

[9] N. H. Bingham, C. M. Goldie, And J. L. Teugels, Regular Variation, Cambridge University Press, Cambridge, UK, 1987.

[10] N. Bleistein and R. A. Handelsman, Asymptotic Expansions of Integrals, Holt, Rinehart and Winston, New York, 1995.

[11] R. Cont And P. Tankov, Financial Modeling with Jump Processes, Chapman and Hall/CRC Press, Boca Raton, FL, 2004.

[12] B. Epstein, Some applications of the Mellin transform in statistics, Ann. Math. Statist., 19 (1948), pp. 370-379.

[13] P. Friz, S. Gerhold, A. Gulisashvili, and S. Sturm, On refined volatility smile expansion in the Heston model, Quant. Finance, 11 (2011), pp. 1151-1164.

[14] K. Gao And R. LeE, Asymptotics of implied volatility to arbitrary order, Finance Stoch., 18 (2014), pp. 349-392.

[15] S. Gerhold, J. F. Morgenbesser, And A. Zrunek, Refined wing asymptotics for the Merton and Kou jump diffusion models, preprint, arXiv:1401.1954v1 [q-fin.PR], 2014; in Proceedings of Advances in Mathematics of Finance -6th General AMaMeF and Banach Center Conference, L. Stettner, ed., 
Banach Center Publications, Warsaw, 2013, to appear.

[16] C. M. Goldie And R. L. Smith, Slow variation with remainder: Theory and applications, Quart. J. Math., 38 (1987), pp. 45-71.

[17] A. Gulisashvili, Analytically Tractable Stochastic Stock Price Models, Springer-Verlag, Berlin, Heidelberg, 2012.

[18] A. Gulisashvili And E. M. Stein, Asymptotic behavior of the stock price distribution density and implied volatility in stochastic volatility models, Appl. Math. Optim., 61 (2010), pp. 287-315.

[19] A. Gulisashvili And J. Vives, Two-sided estimates for distribution densities in models with jumps, in Stochastic Differential Equations and Processes, Springer Proc. Math. 7, M. Zili and D. V. Filatova, eds., Springer-Verlag, Berlin, 2011, pp. 237-252.

[20] S. L. Heston, A closed-form solution for options with stochastic volatility, with applications to bond and currency options, Rev. Financial Stud., 6 (1993), pp. 327-343.

[21] M. Keller-Ressel, Moment explosions and long-term behavior of affine stochastic volatility models, Math. Finance, 21 (2011), pp. 73-98.

[22] S. G. Kou, A jump diffusion model for option pricing, Management Sci., 48 (2002), pp. 1086-1011.

[23] S. G. Kou AND H. WANG, Option pricing under a double exponential jump diffusion model, Management Sci., 50 (2003), pp. 1178-1192.

[24] J. L. Lavoie, T. J. Osler, And R. Tremblay, Fractional derivatives and special functions, SIAM Rev., 18 (1976), pp. 240-268.

[25] P. E. ProtTer, Stochastic Integration and Differential Equations, 2nd ed., Springer-Verlag, Berlin, 2004.

[26] W. Schoutens, Lévy Processes in Finance: Pricing Financial Derivatives, Wiley, Chichester UK, 2003.

[27] R. Wong, Asymptotic Approximations of Integrals, Classics Appl. Math. 34, SIAM, Philadelphia, 2001.

[28] A. Zygmund, Trigonometric Series, Vols. I, II, 3rd ed., Cambridge Mathematical Library, Cambridge University Press, Cambridge, UK, 2002.

[29] A. ZruneK, Volatility Smile Expansions in Lévy Models, Master's thesis, Vienna University of Technology, Vienna, Austria, 2013. 